\title{
Uranium in the Monterey Formation of California
}

\section{U.S. GEOLOGICAL SURVEY BULLETIN 1581-A}



Chapter A

\section{Uranium in the \\ Monterey Formation of California}

By DAVID L. DURHAM

U.S. GEOLOGICAL SURVEY BULLETIN 1581

STUDIES OF URANIUM CONTENT AND GEOCHEMISTRY OF THE MONTEREY FORMATION, CALIFORNIA 


\title{
DEPARTMENT OF THE INTERIOR DONALD PAUL HODEL, Secretary
}

\author{
U.S. GEOLOGICAL SURVEY \\ Dallas L. Peck, Director
}

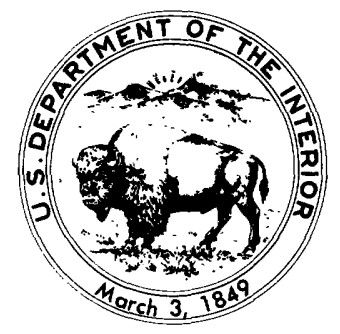

For sale by the

Books and Open-File Reports Section

U.S. Geological Survey

Federal Center

Box 25425

Denver, CO 80225

\section{Library of Congress Cataloging in Publication Data}

Durham, David L., 1925-

Uranium in the Monterey formation of California.

(Studies of uranium content and geochemistry of Monterey formation, California) (U.S. Geological Survey water-supply paper ; 1581-A)

Bibliography: p.

Supt. of Docs. no.: । 19.3:1581-A

1. Uranium ores-California-Pacific Coast. 2. Diagenesis-California-

Pacific Coast. 3. Geology, Stratigraphic-Miocene. 4. Geology-

California_Pacific Coast. 5. Geochemistry-California-Pacific Coast.

I. Title. II. Series. III. Series: Geological Survey bulletin ; 1581-A.

QE75.B9 no. 1581-A

[QE390.2.U7]

$557.3 \mathrm{~s}$

85-600319

$\left[553.4^{\prime} .932^{\prime} 09794\right.$ 


\title{
CONTENTS
}

\author{
Abstract A1 \\ Introduction $\mathbf{A 1}$ \\ Purpose and scope of investigation A1 \\ Fieldwork and acknowledgments A1 \\ Monterey Formation A3 \\ Character $\mathbf{A 3}$ \\ Environment of deposition $\mathbf{A 3}$ \\ Diagenesis A4 \\ Uranium in Monterey Formation A5 \\ Form of uranium $\mathbf{A 5}$ \\ Analytical data A5 \\ Interpretation of analytical data $\mathbf{A 1 7}$ \\ Uranium in the marine environment A22 \\ Sea water A22 \\ Uranium content $\mathbf{A 2 2}$ \\ Extraction of uranium $\mathbf{A 2 2}$ \\ Role of organic carbon A22 \\ Role of phosphorus $\mathbf{A 2 4}$ \\ Role of silica A24 \\ Diatomaceous sediments A25 \\ Summary A25 \\ Conclusions A26 \\ References cited A26
}

\section{FIGURES}

1. Index map of localities $\mathbf{A 2}$

2-7. Histograms showing uranium contents of samples from the Monterey Formation:

2. Outcrop samples A9

3. Outcrop samples from the Temblor Range $\mathbf{A 1 0}$

4. Outcrop samples from outside the Temblor Range A11

5. Samples of porcelanite, porcelaneous mudstone, and cherty porcelanite $\mathbf{A 1 2}$

6. Samples of diatomite and diatomaceous mudstone A13

7. Samples of calcareous mudstone A14

8. Graph showing thorium-uranium ratios for samples from the Monterey Formation A14

9. Typical X-ray diffractograms of porcelanite showing various height-towidth ratios (h/w) of (101) cristobalite peak $\mathbf{A 1 9}$

10. Graph showing relation of uranium content to stage of diagenesis of porcelanite samples A21

11. Graph showing relation of uranium content to organic carbon content of core samples from Monterey Formation and related rocks near Santa Maria A21 


\section{TABLES}

1. Uranium and thorium content of outcrop samples from Monterey Formation A6

2. Summary of uranium analyses of outcrop samples of Monterey Formation $\mathbf{A 8}$

3. Thorium analyses of outcrop samples from Monterey Formation A15

4. Analyses by emission spectroscopy for 19 elements in samples of Monterey Formation that have a wide range of uranium content A15

5. Uranium and thorium content of core samples from the Monterey Formation near the Temblor Range A16

6. Uranium, thorium, and organic carbon content of core samples from the Monterey Formation and related rocks from near Santa Maria A16

7. Uranium and thorium content of diatomite in cores taken on California Continental Borderland A17

8. Uranium and thorium content of lower Miocene diatom ooze from Bering Sea $\mathbf{A 1 7}$

9. Uranium and thorium content of siliceous sediments from the equatorial Pacific A18

10. Uranium and thorium content of siliceous rocks from South America and Mexico A18

11. Height-to-width ratio of the (101) cristobalite peak obtained by X-ray diffractometry of samples of porcelaneous rock 


\title{
Uranium in the Monterey Formation of California
}

\author{
By David L. Durham
}

\begin{abstract}
The Monterey Formation is chiefly marine diatomaceous, porcelaneous, and cherty rocks of Miocene age, which occurs in coastal California. These marine rocks originated as richly diatomaceous sediments that accumulated near the coast in a region of upwelling and high organic productivity, where decomposition of organic matter resulted in anoxic conditions on the sea-floor. Diagenesis that accompanied burial of the diatomaceous sediments converted the biogenous opal of diatoms to cristobalite (opal-CT) and quartz, producing the diatomite, porcelanite, and chert of the Monterey Formation.
\end{abstract}

The uranium content of analyzed samples from the Monterey Formation ranges from less than 2 to more than 1,850 parts per million (ppm). The uranium content of 50 samples collected to represent various kinds of rock in the Monterey, but without regard to their radioactivity in the field, ranges from 1.73 to $83.4 \mathrm{ppm}$, averages $11.1 \mathrm{ppm}$, and has a median value of $6.77 \mathrm{ppm}$. These values are considered representative of the formation.

It is remarkable that even at places in the Temblor Range where the Monterey contains more uranium than it does elsewhere, the only identified uranium minerals are of secondary origin, and they occur in quantities too small to account for the uranium present in the rock. Indeed, most of the uranium in the Monterey is associated with organic material, either by adsorption on the material or by incorporation in organic complexes.

Modern diatomaceous mud, which has accumulated under conditions like those of Monterey deposition, contains more uranium than most sediments. Sea water is an adequate and renewable source for the uranium in the mud, and the anoxic conditions associated with the mud deposition are conducive to the preservation of uranium-rich sediments, if not to their formation.

The Monterey Formation is a potential large-volume, lowgrade uranium source. It also is a potential source of secondary uranium for sandstone-type deposits in other formations.

\section{INTRODUCTION}

\section{Purpose and Scope of the Investigation}

In the mid-1950's low-grade uranium deposits were discovered by prospectors in the Temblor Range (fig. 1) of California (Bowes, 1955, p. 3; Troxel and Morton, 1962, p. 335; Troxel and others, 1957, p. 678; Walker and others, 1956, p. 33-34). Interest in the deposits waned after only a limited amount of exploration and development work had been done, but interest revived briefly in the mid-1960's and again in the mid-1970's when the price of uranium made low-grade deposits more attractive. The Temblor Range uranium deposits occur in siliceous rocks that are commonly assigned to the Monterey Formation and are identical to rocks unequivocally assigned to the Monterey elsewhere. Certain questions concerning uranium in the Monterey Formation come immediately to mind: In what form does the uranium occur? Where did the uranium come from, and when? Are some kinds of rock in the Monterey richer in uranium than are other kinds? Is uranium enrichment of the formation limited to the Temblor Range, or is it more widespread? The purpose of this report is to address these questions by summarizing the character of the Monterey Formation, by reviewing data concerning the origin and diagenetic history of the Monterey, and by reporting and interpreting the results of analyses of samples from the formation. The geochemistry of uranium in the sea is considered, along with modern analogs of the sediments that formed the Monterey.

\section{Fieldwork and Acknowledgments}

Fieldwork was carried out in the Temblor Range in March, April, and October 1977, and elsewhere in October 1978 and April 1980. 


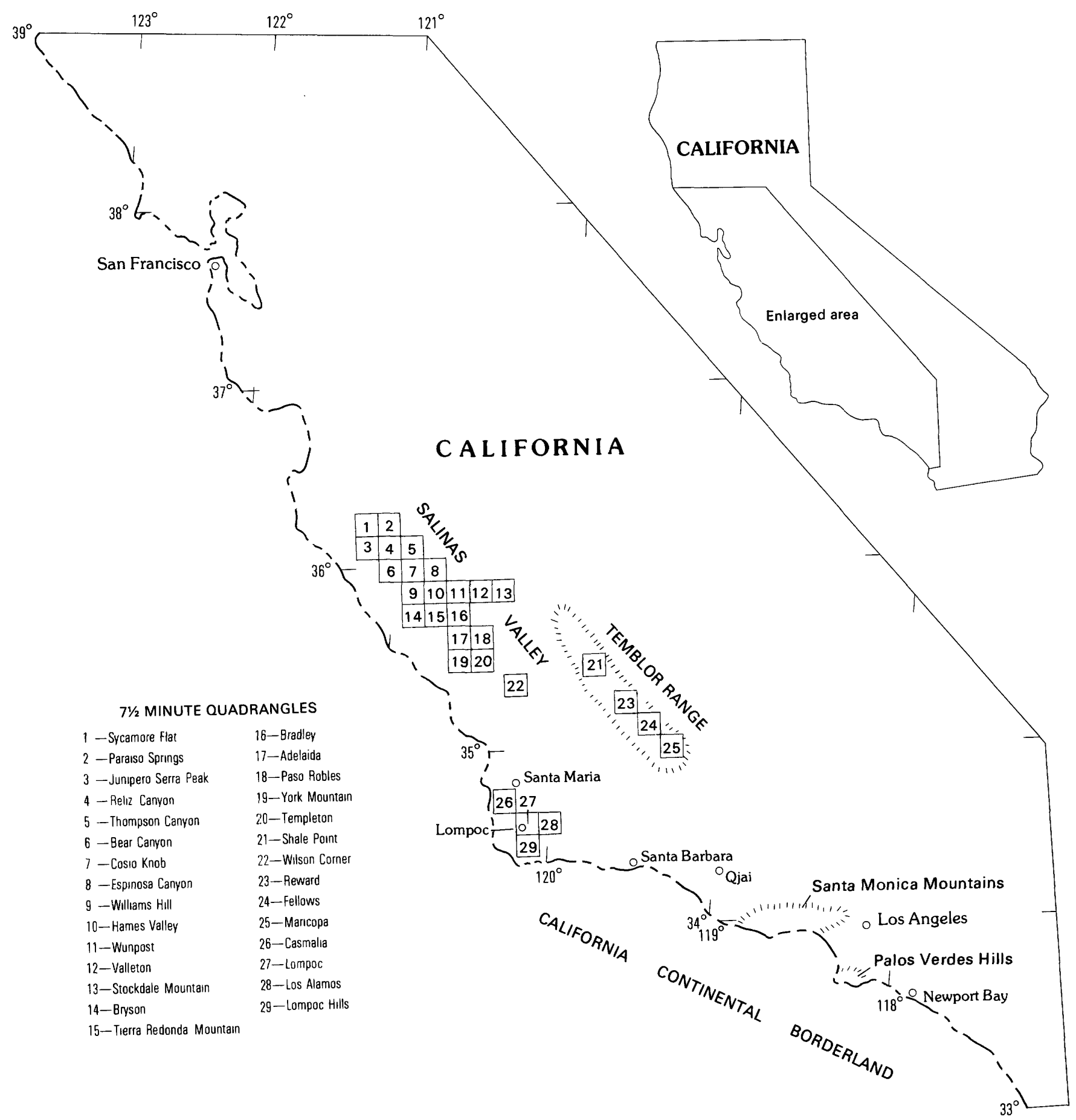

Figure 1. Index map of localities.

Delayed neutron determinations of uranium and thorium were made in the U.S. Geological Survey Analytical Laboratories by H. T. Millard, Jr., R. Bies, C. Bliss, M. F. Coughlin, S. Danahey, A. J. Eartel, C. M. Ellis, B. A. Keaten, R. J. Knight, S. W. Lasater, F. Lumen, C. McFee, R. L. Nelms, C. A. Ramsey, C. L. Shields, M. W. Sold, J. Storey, and R. B. Vaughn;
Harriet Neiman made eU determinations by beta-gamma scaler; M. Retsloff determined elements by emission spectroscopy. Mark Stanton made organic carbon determinations by combustion after acid leaching. Courteney Williamson made X-ray diffractograms of porcelanite samples and determined the height-to-width ratios of the (101) cristobalite peaks on them. G. G. Start made 
X-ray determinations of uranium minerals. J. A. Barron studies fossil diatoms and W. O. Addicott identified fossil mollusks from the Temblor Range. C. M. Isaacs and L. A. Beyer provided core samples. J. R. Hein contributed samples of diatomite from Mexico and South America and core samples of diatomite and diatomaceous sediments from the sea bottom. J. S. Rapp of the California Division of Mines and Geology provided information on uranium claims in the Temblor Range.

\section{MONTEREY FORMATION}

The Monterey Formation contains a variety of rocks, but as so aptly pointed out by Bramlette (1946, p. 2), "its siliceous character makes it one of the most distinctive and easily recognized of the formations in the thick Tertiary System of California." Biogenous opal of diatoms generally is acknowledged as the principal source of the silica in the formation. The Monterey-and correlative units that have local names-occurs in California from just north of San Francisco southward to the Los Angeles area. Some of the best known occurrences are at Newport Bay, in the Palos Verdes Hills, in the Santa Monica Mountains, near Santa Barbara, by Lompoc, near Santa Maria, in the Salinas Valley area, and in the Temblor Range (fig. 1). The thickness of the Monterey is as great as 8,000-10,000 feet in the southern Salinas Valley area (Durham, 1974, p. 32). The formation is marine and of Miocene age. It contains foraminifers representing Kleinpell's (1938) Saucesian, Relizian, Luisian, Mohnian, and Delmontian Stages-in ascending order.

The rocks themselves hold many clues to the origin and history of the Monterey Formation, and presumably also to the origin of the uranium in the unit. The character of the rocks, their environment of deposition, and their diagenetic history all are important to an understanding of the uranium content of the Monterey.

\section{Character}

The Monterey Formation is made up mainly of diatomaceous, porcelaneous, and cherty rocks. Where diatomite and diatomaceous mudstone occur they are in the upper part of the formation-as near Lompoc, in the Temblor Range, and in parts of the Salinas Valley. Porcelanite and porcelaneous mudstone generally constitute the bulk of the upper part of the Monterey; and cherty rocks, siliceous mudstone, and calcareous mudstone are more characteristic of the lower part of the unit. Even the calcareous mudstone has a high silica content. Dolomite beds and concretions occur throughout the formation, and locally the Monterey contains beds of vitric tuff and beds of pelletal phosphorite. Rocks of the Monterey commonly are thin bedded or laminated, but at some places they are thick bedded or massive.

The most common rocks of the formation have a high silica content. For example, ten samples of diatomite and porcelaneous rocks analyzed by emission spectroscopy had 31-39 percent silicon (table 4). Analyses of siliceous Monterey rocks given by Bramlette (1946, p. 13) showed about 70-90 percent $\mathrm{SiO}_{2}$. X-ray analysis reveals that the silica is mainly in the form of opal, cristobalite, and quartz. The rocks also contain an appreciable amount of organic carbon (table 6).

The lithologic character of the Monterey Formation places certain restraints on the origin of the unit. Any reconstruction of the conditions under which the Monterey formed must account for, among other things, the silica and organic-carbon content of the rock, the general lack of all but the finest clastic material, the common lamination of the rock, and the occurrence of phosphorite beds.

\section{Environment of Deposition}

The abundance of remains of marine organisms in the Monterey Formation demonstrates the marine origin of the unit. Investigations of fossil foraminifers (Arnal and Vedder, 1976; Bandy and Arnal, 1969; Bandy and Kolpack, 1963; Ingle, 1963b, 1967, 1972, 1973; Patet, 1972; Smith, 1968), diatoms (Barron, 1973, 1975), fish (David, 1940a, 1943; Pierce, 1956), radiolarians (Casey and Price, 1973; Ingle, 1967), and silicoflagellates (Cornell, 1969; Mandra, 1959) allow the following generalizations concerning the environment of deposition of the Monterey:

(1) The sediments accumulated in basins generally near shore.

(2) Water depths and effective sill depths of the basins of deposition varied, but commonly were bathyal.

(3) The region was one of upwelling and high organic productivity.

(4) Bottom water was anoxic much of the time.

(5) The contribution of terrigenous clastic material was minor in areas of typical Monterey sedimentation.

(6) Water temperatures fluctuated, perhaps reflecting changes in current patterns, but probably were comparable generally to water temperatures off California today.

The conditions under which sediments of the Monterey Formation accumulated commonly are likened to conditions presently found on the California Continental Borderland and in parts of the Gulf of California (Ingle, 1963a, b, p. 1771; Bandy and Kolpack, 1963, p. 154). Brongersma-Sanders (1948, p. 5, 1951, p. 406) compared the sediments that formed the Monterey to those found today in Walvis Bay off southwest Africa, 
and noted (1957, p. 970) that the catastrophic mass killing of fish recorded in a bed of diatomite near Lompoc is the exact equivalent of a layer of fish deposited in Walvis Bay during an enormous mass extinction of fish in 1924. Bramlette (1946, p. 37-38) concluded that diatomaceous deposits of the Monterey appear to be due to a combination of high productivity of diatoms and relatively little deposition of clastic material. Brongersma-Sanders (1957, p. 970) considered that the open-sea conditions indicated by fossil fish in the Monterey, together with the presence of diatom ooze, demonstrates deposition in a region of upwelling and hypertorphic conditions. Lipps and Kalisky (1973) studied calcareous nannoplankton in Oligocene and Miocene rocks and concluded that (1) when the Monterey was deposited, the California coast was under the influence of a current that flowed south with a velocity nearly that of the modern California Current, and (2) upwelling probably was as important in the region during the Miocene as it is today.

In modern seas, areas of richly diatomaceous sediments occur where divergence of surface-water masses causes upwelling of deep waters rich in nutrients, especially silica (Brongersma-Sanders, 1948, p. 82; Calvert, 1974, p. 278; Lisitsyn, 1967, p. 998; Ramsay, 1973, p. 199). Upwelling conditions may persist in an area for long periods of time. For example, Siesser $(1980$, p. 285) determined that the upwelling system off southwest Africa began about 10 million years ago, and Busch and Keller (1981, p. 707) noted that upwelling has continued off the coast of Peru since at least the late Miocene.

Conditions under which modern marine diatomaceous sediments accumulate permits the following generalizations, which can be compared to conditions already outlined for deposition of the Monterey Formation:

(1) Sustained upwelling of nutrient-rich waters to the surface and consequent prolific production in the euphotic zone is required (Brongersma-Sanders, 1948, p. 82; Calvert, 1974, p. 278; Lisitsyn, 1967, p. 998 , Ramsay, 1973, p. 199).

(2) Turbulent water and strong bottom currents must be absent to allow settling of very fine grained material (Baturin, 1971b, p. 374; Lisitsyn, 1967, p. 1125).

(3) Sedimentation of diatoms is favored by production of robust forms (Brongersma-Sanders, 1948, p. 83; Lisitsyn, 1967, p. 999), and by incorporation of the diatoms in fecal pellets (Lisitsyn, 1972, p. 154; Schrader, 1971, p. 57; Smayda, 1971, p. 117).

(4) Little or no terrigenous clastic detritus or other material dilutes present-day diatomaceous deposits (Brongersma-Sanders, 1948, p. 82-83; Lisitsyn, 1967, p. 1121).
(5) Rapid sedimentation favors preservation of organic carbon (Muller and Suess, 1979, p. 1356), and decomposition of organic material in the sediments by sulfate-reducing bacteria produces $\mathrm{H}_{2} \mathrm{~S}$ (Brongersma-Sanders, 1948, p. 89, 1966, p. 41; Berner, 1978, p. 493; Boon and others, 1978, p. 638; Redfield and others, 1963, p. 42).

The anoxic conditions commonly associated with diatomaceous sediments are a consequence of, not a prerequisite for the accumulation of the sediments. However, because of the importance of a reducing environment in the geochemistry of uranium, the accumulation of diatomaceous sediments under anoxic conditions seems to be an important factor affecting uranium content of the sediments.

\section{Diagenesis}

Studies of the diagenesis of the Monterey Formation and similar rocks (Calvert, 1966; Hein and others, 1978; Iijima and Tada, 1981; Isaacs, 1979; Kastner and others, 1977; Murata and others, 1977; Murata and Larson, 1975; Murata and Nakata, 1974; Siever and Stein, 1976) lead to the following generalizations concerning the formation:

(1) The Monterey began as richly diatomaceous sediments composed mainly of biogenous opal (opalA) extracted by diatoms from normal sea water to form their frustules.

(2) Early diagenesis of the sediments involved dissolution of the biogenous opal followed by precipitation of either inorganic opal or highly disordered cryptocrystalline cristobalite (opal-CT).

(3) Diagenesis continued with progressive ordering of cristobalite by a solid-state transformation.

(4) In the final stage of diagenesis, well-ordered cristobalite was converted to microcrystalline quartz, probably by a solution-precipitation process involving only limited migration of silica.

(5) The conversion of opal to cristobalite and quartz is a function mainly of temperature; the conversion also is affected by the purity of the diatomaceous sediments and the chemical nature of interstitial water.

(6) The cristobalite found in outcrops of Monterey rocks may be partly of secondary origin - that is, added during or after uplift of the rocks. 


\section{URANIUM IN THE MONTEREY FORMATION}

\section{Form of Uranium}

Autunite, meta-autunite, and uranophane were identified by X-ray diffractometry in samples of Monterey Formation from the Temblor Range. Carnotite is the only other uranium mineral reported from the area (Finch, 1967, p. 8). The identified uranium minerals are all secondary and are found on fracture and bedding surfaces. The concentration of uranium in fractured rock and fault zones is mentioned in descriptions of uranium deposits of the Temblor Range (Walker and others, 1956, p. 33; Troxel and others, 1957, p. 687). Finch $(1967$, p. 8) classified the Temblor Range deposits as epigenetic-that is, deposits formed by precipitation from solutions that moved through the host rock-and Osterwald (1965, p. 125) noted structural control of uranium mineralization at a claim in the Temblor Range. Obviously, then, the association of secondary uranium minerals with zones of broken rock is related to the ease with which uraniferous ground water can permeate such zones. But some samples contain much more uranium than can be accounted for by any secondary uranium minerals present. One sample (764005), for example, contained more than 1850 parts per million ( $\mathrm{ppm}$ ) uranium in rock almost devoid of identifiable uranium minerals. No uranium minerals were seen nor were any found by $\mathrm{X}$-ray analysis in Monterey rocks outside the Temblor Range. Clearly, secondary uranium minerals do not account for all or even for very much of the uranium present in the Monterey Formation.

Langmuir (1978, p. 562) noted that autunite and uranophane most often occur near uraniferous alkalic igneous rocks, which tend to be low in vanadium and relatively high in silica or phosphate, or both. The term "alkalic igneous rocks" might be replaced in this statement by the term "rocks of the Monterey Formation." The notion that autunite precipitates from ground water where phosphate is present and vanadium rare and that uranophane forms in silica-rich systems deficient in vanadium and phosphate can apply as well to Monterey terrains as to igneous terrains. The abundance of secondary uranium minerals in the Temblor Range compared to any other areas of Monterey outcrops perhaps can be attributed to the effect of the aridity of the Temblor Range on the vadose-water regime there.

Five samples (764005, 764010, 771020, 772027, 802037) from the Monterey Formation, which did not have any identifiable minerals, nevertheless did contain from 50 to $1850 \mathrm{ppm}$ uranium. Radioluxographs of these samples show a diffuse background radioactivity. The only concentrations of radioactivity were related to cracks and to iron oxide discoloration in the rock. The scanning electron microscope failed to show any identifiable uranium minerals even in the sample (764005) that contained more than $1850 \mathrm{ppm}$ uranium (J. R. Hein, personal commun., 1981).

\section{Analytical Data}

Delayed neutron analysis of 111 outcrop samples from the Monterey Formation were made for uranium and thorium (table 1). Table 2 summarizes the data, and figures 2-7 show it graphically. All results given in the tables are in the form reported from the laboratory.

The uranium content of the 111 samples analyzed (fig. 2) ranged from about 1.7 to $1854 \mathrm{ppm}^{1}$, averaged $54.12 \mathrm{ppm}$, and had a median value of $10.4 \mathrm{ppm}$, but these numbers have little meaning beyond showing that the Monterey Formation seems to have a higher uranium content than do most rocks-certainly more than the average of 3-4 ppm uranium for all shale (Swanson, 1961, p. 70). The results of analyses of particular categories of samples were more revealing. Samples from the Temblor Range (fig. 3) had a higher average and median value than did other Monterey Formation samples, but this mainly was because of the high uranium content of samples from uranium prospects. Uranium values for samples from the Temblor Range, but not from prospects, were much closer to those for samples collected outside the range. Perhaps more significant are the results of analyses of 50 samples collected to represent different kinds of rock within the Monterey Formation and without bias with regard to their radioactivity as measured in the field with a scintillation counter (fig. $4 B$ ). The uranium content of these 50 samples ranged from 1.73 to $83.4 \mathrm{ppm}$, had an average value of $11.1 \mathrm{ppm}$, and had a median value of $6.77 \mathrm{ppm}$; these values probably can be considered representative of a large part of the Monterey throughout California, and they too show that the Monterey as a whole has a higher uranium content than do most formations.

Consideration of the uranium content of various kinds of rock in the Monterey Formation also is useful. The uranium content of 64 samples of porcelanite, the most common rock in the Monterey, ranged from 2.40 to $767 \mathrm{ppm}$, had an average value of 48.41 , and had a median value of $10.8 \mathrm{ppm}$ (fig. 5), but these figures are

\footnotetext{
${ }^{1}$ Another analysis of one sample, 764005 , showed $2380 \mathrm{ppm}$ uranium. The reason for the discrepancy is unknown.
} 
Table 1. Uranium and thorium content of outcrop samples from Monterey Formation

[Counties: K, Kern; M, Monterey; SB, Santa Barbara; SLO, San Luis Obispo. Abbreviations for rocks: ch, chert; di, diatomite; do, dolomite; $\mathrm{mc}$, calcareous mudstone; md, diatomaceous mudstone; mp, porcelaneous mudstone; ms, siliceous mudstone; pc, cherty porcelanite; ph, phosphorite; po, porcelanite; ss, sandstone; tu, tuff. ---, leaders indicate no data]

\begin{tabular}{|c|c|c|c|c|c|c|c|c|c|c|c|}
\hline $\begin{array}{l}\text { Field } \\
\text { No. }\end{array}$ & Lab. No. & Lat. N & Long. W & $\begin{array}{l}\text { Quadrangle } \\
(71 / 2 \mathrm{~min})\end{array}$ & County & Rock & $\begin{array}{l}\text { Scintill- } \\
\text { ometer } \\
\text { reading } \\
\text { in field } \\
\text { (c.p.s.) }\end{array}$ & $\underset{(p p m)}{U}$ & $\begin{array}{l}\text { Th } \\
\text { (ppm) }\end{array}$ & $\underset{(p p m)}{\mathrm{eU}}$ & $T h / U$ \\
\hline $\begin{array}{c}1764001 \mathrm{~A} \\
1_{764001 \mathrm{~B}} \\
1_{764002} \\
764003 \\
1764005\end{array}$ & $\begin{array}{l}\text { D187770 } \\
\text { D187771 } \\
\text { D187772 } \\
\text { D187773 } \\
\text { D187774 }\end{array}$ & $\begin{array}{l}35^{\circ} 05^{\prime} 55^{\prime \prime} \\
35^{\circ} 05^{\prime} 55^{\prime \prime} \\
35^{\circ} 05^{\prime} 45^{\prime \prime} \\
35^{\circ} 05^{\prime} 35^{\prime \prime} \\
35^{\circ} 08^{\prime} 05^{\prime \prime}\end{array}$ & $\begin{array}{l}119^{\circ} 28^{\prime} 40^{\prime \prime} \\
119^{\circ} 8^{\prime} 40^{\prime \prime} \\
119^{\circ} 28^{\prime} 05^{\prime \prime} \\
119^{\circ} 28^{\prime} 25^{\prime \prime} \\
119^{\circ} 31^{\prime} 25^{\prime \prime}\end{array}$ & $\begin{array}{l}\text { Maricopa } \\
\text { Maricopa } \\
\text { Maricopa } \\
\text { Maricopa } \\
\text { Fellows }\end{array}$ & $\begin{array}{l}\mathbf{K} \\
\mathbf{K} \\
\mathbf{K} \\
\mathbf{K} \\
\mathbf{K}\end{array}$ & $\begin{array}{l}\text { po } \\
\text { po } \\
\text { po } \\
\text { po } \\
\text { di }\end{array}$ & $\begin{array}{c}630 \\
580 \\
800-900 \\
200 \\
6000\end{array}$ & $\begin{array}{r}165.67 \\
51.51 \\
205.48 \\
4.37 \\
1854.73\end{array}$ & $\begin{array}{c}--- \\
--- \\
-02 \\
---\end{array}$ & $\begin{array}{r}100 \\
40 \\
120 \\
20 \\
1220\end{array}$ & $\begin{array}{c}--- \\
-- \\
1.38 \\
---\end{array}$ \\
\hline $\begin{array}{l}{ }_{1}^{1} 764006 \\
1764007 \\
{ }^{1} 764008 \\
{ }^{1} 764009 \\
{ }^{1} 764010\end{array}$ & $\begin{array}{l}\text { D187775 } \\
\text { D187776 } \\
\text { D187777 } \\
\text { D187778 } \\
\text { D187779 }\end{array}$ & $\begin{array}{l}35^{\circ} 10^{\prime} 25^{\prime \prime} \\
35^{\circ} 10^{\prime} 30^{\prime \prime} \\
35^{\circ} 11^{\prime} 25^{\prime \prime} \\
35^{\circ} 20^{\prime} 50^{\prime \prime} \\
35^{\circ} 35^{\prime} 35^{\prime \prime}\end{array}$ & $\begin{array}{l}19^{\circ} 35^{\prime} 10^{\prime \prime} \\
119^{\circ} 35^{\prime} 05^{\prime \prime} \\
119^{\circ} 36^{\prime} 20^{\prime \prime} \\
119^{\circ} 42^{\prime} 10^{\prime \prime} \\
19^{\circ} 59^{\prime} 30^{\prime \prime}\end{array}$ & $\begin{array}{l}\text { Fellows } \\
\text { Fellows } \\
\text { Fellows } \\
\text { Reward } \\
\text { Shale Point }\end{array}$ & $\begin{array}{c}\text { SLO } \\
\text { SLO } \\
\mathbf{K} \\
\mathbf{K} \\
\mathbf{K}\end{array}$ & $\begin{array}{l}\text { di } \\
\text { di } \\
\text { po } \\
\text { po } \\
\text { po }\end{array}$ & $\begin{array}{c}300-350 \\
1800 \\
2500 \\
1800 \\
600\end{array}$ & \begin{tabular}{r|}
31.83 \\
290.21 \\
259.78 \\
149.00 \\
83.13
\end{tabular} & $\begin{array}{l}--- \\
--- \\
--- \\
---\end{array}$ & $\begin{array}{r}30 \\
240 \\
200 \\
150 \\
60\end{array}$ & $\begin{array}{l}--- \\
--- \\
--- \\
-- \\
--\end{array}$ \\
\hline $\begin{array}{r}771001 \\
771008 \\
771010 \\
1771016 \\
771020\end{array}$ & $\begin{array}{l}\text { M131628 } \\
\text { M131629 } \\
\text { M131630 } \\
\text { M131631 } \\
\text { M131632 }\end{array}$ & $\begin{array}{l}35^{\circ} 11^{\prime} 35^{\prime \prime} \\
35^{\circ} 11^{\prime} 45^{\prime \prime} \\
35^{\circ} 11^{\prime} 40^{\prime \prime} \\
35^{\circ} 11^{\prime} 50^{\prime \prime} \\
35^{\circ} 11^{\prime} 30^{\prime \prime}\end{array}$ & $\begin{array}{l}119^{\circ} 35^{\prime} 55^{\prime \prime} \\
119^{\circ} 36^{\prime} 20^{\prime \prime} \\
119^{\circ} 36^{\prime} 40^{\prime \prime} \\
119^{\circ} 37^{\prime} 05^{\prime \prime} \\
119^{\circ} 36^{\prime} 50^{\prime \prime}\end{array}$ & $\begin{array}{l}\text { Fellows } \\
\text { Fellows } \\
\text { Fellows } \\
\text { Fellows } \\
\text { Fellows }\end{array}$ & $\begin{array}{l}\mathbf{K} \\
\mathbf{K} \\
\mathbf{K} \\
\mathbf{K} \\
\mathbf{K}\end{array}$ & $\begin{array}{c}\text { di } \\
\text { di } \\
\mathrm{mp} \\
\text { di } \\
\text { di }\end{array}$ & $\begin{array}{c}160-180 \\
110-130 \\
160-180 \\
250 \\
120-130\end{array}$ & $\begin{array}{c}10.4 \\
4.44 \\
20.2 \\
44.2 \\
12.2\end{array}$ & $\begin{array}{c}10 . \\
--- \\
--- \\
---\end{array}$ & $\begin{array}{l}20 \\
10 \\
30 \\
50 \\
10\end{array}$ & $\begin{array}{c}0.96 \\
--- \\
--- \\
--- \\
--\end{array}$ \\
\hline $\begin{array}{r}1771021 \\
771040 \\
771051 \\
1771064 \\
1771065\end{array}$ & $\begin{array}{l}\text { M131633 } \\
\text { M131634 } \\
\text { M131635 } \\
\text { M131636 } \\
\text { M131637 }\end{array}$ & $\begin{array}{l}35^{\circ} 11^{\prime} 30^{\prime \prime} \\
35^{\circ} 11^{\prime} 25^{\prime \prime} \\
35^{\circ} 10^{\prime} 30^{\prime \prime} \\
35^{\circ} 10^{\prime} 40^{\prime \prime} \\
35^{\circ} 11^{\prime} 25^{\prime \prime}\end{array}$ & $\begin{array}{l}119^{\circ} 36^{\prime} 55^{\prime \prime} \\
119^{\circ} 36^{\prime} 15^{\prime \prime} \\
119^{\circ} 36^{\prime} 45^{\prime \prime} \\
119^{\circ} 37^{\prime} 05^{\prime \prime} \\
119^{\circ} 37^{\prime} 00^{\prime \prime}\end{array}$ & $\begin{array}{l}\text { Fellows } \\
\text { Fellows } \\
\text { Fellows } \\
\text { Fellows } \\
\text { Fellows }\end{array}$ & $\begin{array}{c}\mathbf{K} \\
\mathbf{K} \\
\text { SLO } \\
\text { SLO } \\
\mathbf{K}\end{array}$ & $\begin{array}{l}\mathrm{mp} \\
\mathrm{mp} \\
\text { po } \\
\text { po } \\
\text { po }\end{array}$ & $\begin{array}{c}1100-1200 \\
140-160 \\
300-600 \\
800-900 \\
2700\end{array}$ & $\begin{array}{c}86.6 \\
3.10 \\
20.1 \\
71.5 \\
344 .\end{array}$ & $\begin{array}{l}--- \\
5.5 \\
-- \\
-- \\
--\end{array}$ & $\begin{array}{r}120 \\
10 \\
30 \\
120 \\
250\end{array}$ & $\begin{array}{l}--- \\
1.77 \\
--- \\
--- \\
---\end{array}$ \\
\hline $\begin{array}{r}771080 \\
771083 \\
1771120 \\
771139 \\
772009\end{array}$ & $\begin{array}{l}\text { M131638 } \\
\text { M131639 } \\
\text { M131640 } \\
\text { M131641 } \\
\text { M131642 }\end{array}$ & $\begin{array}{l}35^{\circ} 11^{\prime} 25^{\prime \prime} \\
35^{\circ} 11^{\prime} 25^{\prime \prime} \\
35^{\circ} 10^{\prime} 45^{\prime \prime} \\
35^{\circ} 10^{\prime} 05^{\prime \prime} \\
35^{\circ} 09^{\prime} 20^{\prime \prime}\end{array}$ & $\begin{array}{l}119^{\circ} 36^{\prime} 20^{\prime \prime} \\
119^{\circ} 34^{\prime} 50^{\prime \prime} \\
119^{\circ} 35^{\prime} 45^{\prime \prime} \\
119^{\circ} 34^{\prime} 45^{\prime \prime} \\
119^{\circ} 34^{\prime} 50^{\prime \prime}\end{array}$ & $\begin{array}{l}\text { Fellows } \\
\text { Fellows } \\
\text { Fellows } \\
\text { Fellows } \\
\text { Fellows }\end{array}$ & $\begin{array}{l}\text { SLO } \\
\mathbf{K} \\
\mathbf{K} \\
\text { SLO } \\
\text { SLO }\end{array}$ & $\begin{array}{c}\text { po } \\
\text { po } \\
\text { di } \\
\text { md } \\
\text { po }\end{array}$ & $\begin{array}{c}120-130 \\
250 \\
190 \\
180-220 \\
200-230\end{array}$ & $\begin{array}{l}9.34 \\
55.3 \\
33.6 \\
27.4 \\
14.3\end{array}$ & $\begin{array}{l}10 . \\
-- \\
15 .\end{array}$ & $\begin{array}{l}20 \\
50 \\
30 \\
40 \\
30\end{array}$ & $\begin{array}{r}1.07 \\
-- \\
-- \\
-- \\
1.05\end{array}$ \\
\hline $\begin{array}{l}1772015 \\
1772027 \\
1772031 \\
1772035 \\
772054 \mathrm{~A}\end{array}$ & $\begin{array}{l}\text { M131643 } \\
\text { M131644 } \\
\text { M131645 } \\
\text { M131646 } \\
\text { M131647 }\end{array}$ & $\begin{array}{l}35^{\circ} 08^{\prime} 50^{\prime \prime} \\
35^{\circ} 10^{\prime} 15^{\prime \prime} \\
35^{\circ} 10^{\prime} 35^{\prime \prime} \\
35^{\circ} 10^{\prime} 05^{\prime \prime} \\
35^{\circ} 10^{\prime} 25^{\prime \prime}\end{array}$ & $\begin{array}{l}119^{\circ} 35^{\prime} 00^{\prime \prime} \\
119^{\circ} 37^{\prime} 05^{\prime \prime} \\
119^{\circ} 37^{\prime} 10^{\prime \prime} \\
119^{\circ} 36^{\prime} 40^{\prime \prime} \\
119^{\circ} 35^{\prime} 15^{\prime \prime}\end{array}$ & $\begin{array}{l}\text { Fellows } \\
\text { Fellows } \\
\text { Fellows } \\
\text { Fellows } \\
\text { Fellows }\end{array}$ & $\begin{array}{l}\text { SLO } \\
\text { SLO } \\
\text { SLO } \\
\text { SLO } \\
\text { SLO }\end{array}$ & $\begin{array}{l}\text { po } \\
\text { po } \\
\text { po } \\
\text { po } \\
\text { po }\end{array}$ & $\begin{array}{c}250-300 \\
600-660 \\
1300 \\
1000-1200 \\
130-150\end{array}$ & $\begin{array}{c}21.1 \\
767 . \\
109 . \\
131 . \\
21.9\end{array}$ & $\begin{array}{l}--- \\
--- \\
--- \\
---\end{array}$ & $\begin{array}{r}40 \\
390 \\
110 \\
130 \\
20\end{array}$ & $\begin{array}{l}--- \\
-- \\
--- \\
--\end{array}$ \\
\hline $\begin{array}{r}772063 \\
772083 \\
1772111 \\
772113 \\
1772115\end{array}$ & $\begin{array}{l}\text { M131648 } \\
\text { M131649 } \\
\text { M131650 } \\
\text { M131651 } \\
\text { M131652 }\end{array}$ & $\begin{array}{l}35^{\circ} 12^{\prime} 05^{\prime \prime} \\
35^{\circ} 11^{\prime} 25^{\prime \prime} \\
35^{\circ} 08^{\prime} 25^{\prime \prime} \\
35^{\circ} 07^{\prime} 45^{\prime \prime} \\
35^{\circ} 07^{\prime} 45^{\prime \prime}\end{array}$ & $\begin{array}{l}119^{\circ} 37^{\prime} 00^{\prime \prime} \\
119^{\circ} 34^{\prime} 50^{\prime \prime} \\
119^{\circ} 33^{\prime} 10^{\prime \prime} \\
119^{\circ} 31^{\prime} 15^{\prime \prime} \\
119^{\circ} 31^{\prime} 30^{\prime \prime}\end{array}$ & $\begin{array}{l}\text { Fellows } \\
\text { Fellows } \\
\text { Fellows } \\
\text { Fellows } \\
\text { Fellows }\end{array}$ & $\begin{array}{l}\mathbf{K} \\
\mathbf{K} \\
\mathbf{K} \\
\mathbf{K} \\
\mathbf{K}\end{array}$ & $\begin{array}{l}\text { di } \\
\text { di } \\
\text { po } \\
\text { di } \\
\text { di }\end{array}$ & $\begin{array}{r}90-100 \\
120-130 \\
230-250 \\
100-120 \\
200-210\end{array}$ & $\begin{array}{l}5.46 \\
2.58 \\
74.3 \\
44.2 \\
18.6\end{array}$ & $\begin{array}{r}--- \\
10.9 \\
--- \\
--- \\
---\end{array}$ & $\begin{array}{l}10 \\
10 \\
50 \\
20 \\
20\end{array}$ & $\begin{array}{c}--- \\
4.22 \\
--- \\
--\end{array}$ \\
\hline $\begin{array}{l}772122 \\
772139 \\
772140 \\
772149 \\
773009\end{array}$ & $\begin{array}{l}\text { M131653 } \\
\text { M131654 } \\
\text { M131655 } \\
\text { M131656 } \\
\text { D192877 }\end{array}$ & $\begin{array}{l}35^{\circ} 08^{\prime} 00^{\prime \prime} \\
35^{\circ} 08^{\prime} 05^{\prime \prime} \\
35^{\circ} 08^{\prime} 15^{\prime \prime} \\
35^{\circ} 08^{\prime} 00^{\prime \prime} \\
36^{\circ} 10^{\prime} 30^{\prime \prime}\end{array}$ & $\begin{array}{l}119^{\circ} 33^{\prime} 55^{\prime \prime} \\
119^{\circ} 32^{\prime} 30^{\prime \prime} \\
119^{\circ} 32^{\prime} 25^{\prime \prime} \\
119^{\circ} 32^{\prime} 15^{\prime \prime} \\
121^{\circ} 13^{\prime} 40^{\prime \prime}\end{array}$ & $\begin{array}{l}\text { Fellows } \\
\text { Fellows } \\
\text { Fellows } \\
\text { Fellows } \\
\text { Thompson Canyon }\end{array}$ & $\begin{array}{c}\text { SLO } \\
\mathbf{K} \\
\mathbf{K} \\
\mathbf{K} \\
\mathbf{M}\end{array}$ & $\begin{array}{l}\text { po } \\
\text { po } \\
\text { di } \\
\text { di } \\
\text { po }\end{array}$ & $\begin{array}{c}110-120 \\
180-210 \\
180-220 \\
220 \\
---\end{array}$ & $\begin{array}{c}4.40 \\
7.99 \\
77.9 \\
22.2 \\
3.28\end{array}$ & $\begin{array}{l}--- \\
--- \\
--- \\
-- \\
--\end{array}$ & $\begin{array}{l}10 \\
10 \\
50 \\
30 \\
10\end{array}$ & $\begin{array}{l}-- \\
-- \\
-- \\
--\end{array}$ \\
\hline $\begin{array}{l}773010 \\
784001 \\
784002 \\
784003 \\
784004\end{array}$ & $\begin{array}{l}\text { D192878 } \\
\text { M137998 } \\
\text { M137999 } \\
\text { M138000 } \\
\text { M138001 }\end{array}$ & $\begin{array}{l}36^{\circ} 10^{\prime} 00^{\prime \prime} \\
34^{\circ} 36^{\prime} 00^{\prime \prime} \\
34^{\circ} 36^{\prime} 00^{\prime \prime} \\
34^{\circ} 37^{\prime} 10^{\prime \prime} \\
34^{\circ} 37^{\prime} 20^{\prime \prime}\end{array}$ & $\begin{array}{l}121^{\circ} 11^{\prime} 55^{\prime \prime} \\
120^{\circ} 27^{\prime} 45^{\prime \prime} \\
120^{\circ} 27^{\prime} 45^{\prime \prime} \\
120^{\circ} 24^{\prime} 05^{\prime \prime} \\
120^{\circ} 23^{\prime} 55^{\prime \prime}\end{array}$ & $\begin{array}{l}\text { Thompson Canyon } \\
\text { Lompoc Hills } \\
\text { Lompoc Hills } \\
\text { Lompoc Hills } \\
\text { Lompoc Hills }\end{array}$ & $\begin{array}{l}\text { M } \\
\text { SB } \\
\text { SB } \\
\text { SB } \\
\text { SB }\end{array}$ & $\begin{array}{l}\mathrm{mp} \\
\mathrm{di} \\
\text { ch } \\
\text { po } \\
\text { di }\end{array}$ & $\begin{array}{c}-- \\
90-110 \\
70-90 \\
200-220 \\
70-80\end{array}$ & $\begin{array}{c}5.81 \\
8.40 \\
3.21 \\
18.5 \\
2.26\end{array}$ & $\begin{array}{r}<7.6 \\
<2.8 \\
<7.3 \\
8.2\end{array}$ & $\begin{array}{l}<10 \\
-- \\
--- \\
--\end{array}$ & $\begin{array}{r}--- \\
<0.90 \\
<0.87 \\
<0.39 \\
3.63\end{array}$ \\
\hline $\begin{array}{l}784005 \\
784006 \\
784007 \\
784008 \\
784009\end{array}$ & $\begin{array}{l}\text { M138002 } \\
\text { M138003 } \\
\text { M138004 } \\
\text { M138005 } \\
\text { M138006 }\end{array}$ & $\begin{array}{l}34^{\circ} 42^{\prime} 20^{\prime \prime} \\
34^{\circ} 44^{\prime} 00^{\prime \prime} \\
34^{\circ} 49^{\prime} 05^{\prime \prime} \\
35^{\circ} 28^{\prime} 15^{\prime \prime} \\
35^{\circ} 28^{\prime} 15^{\prime \prime}\end{array}$ & $\begin{array}{l}120^{\circ} 17^{\prime} 00^{\prime \prime} \\
120^{\circ} 26^{\prime} 20^{\prime \prime} \\
120^{\circ} 31^{\prime} 30^{\prime \prime} \\
120^{\circ} 22^{\prime} 20^{\prime \prime} \\
120^{\circ} 22^{\prime} 20^{\prime \prime}\end{array}$ & $\begin{array}{l}\text { Los Alamos } \\
\text { Lompoc } \\
\text { Casmalia } \\
\text { Wilson Corner } \\
\text { Wilson Corner }\end{array}$ & $\begin{array}{l}\text { SB } \\
\text { SB } \\
\text { SB } \\
\text { SLO } \\
\text { SLO }\end{array}$ & $\begin{array}{l}\mathrm{mp} \\
\mathrm{di} \\
\mathrm{di} \\
\mathrm{po} \\
\mathrm{ph}\end{array}$ & $\begin{array}{c}110-120 \\
120-130 \\
80-90 \\
160-180 \\
160-180\end{array}$ & $\begin{array}{c}11.5 \\
7.65 \\
3.58 \\
10.7 \\
39.8\end{array}$ & $\begin{array}{r}<5.2 \\
<6.5 \\
6.8 \\
<5.3 \\
<8.8\end{array}$ & $\begin{array}{l}--- \\
-- \\
-- \\
--\end{array}$ & $\begin{array}{r}<0.45 \\
<0.85 \\
1.90 \\
<0.50 \\
<0.72\end{array}$ \\
\hline $\begin{array}{l}784010 \\
784011\end{array}$ & $\begin{array}{l}\text { M138007 } \\
\text { M138008 }\end{array}$ & $\begin{array}{l}35^{\circ} 59^{\prime} 10^{\prime \prime} \\
35^{\circ} 59^{\prime} 10^{\prime \prime}\end{array}$ & $\begin{array}{l}121^{\circ} 00^{\prime} 30^{\prime \prime} \\
121^{\circ} 00^{\prime} 40^{\prime \prime}\end{array}$ & $\begin{array}{l}\text { Williams Hill } \\
\text { Williams Hill }\end{array}$ & $\begin{array}{l}\mathbf{M} \\
\mathbf{M}\end{array}$ & $\begin{array}{l}\mathrm{mp} \\
\mathrm{mp}\end{array}$ & $\begin{array}{l}420-470 \\
220-250\end{array}$ & $\begin{array}{l}55.4 \\
18.9\end{array}$ & $\begin{array}{l}<12 . \\
<6.8\end{array}$ & --- & $\begin{array}{l}<0.22 \\
<0.36\end{array}$ \\
\hline
\end{tabular}

\footnotetext{
${ }^{1}$ Sample from uranium prospect.
} 
Table 1. Uranium and thorium content of outcrop samples from Monterey Formation-Continued

\begin{tabular}{|c|c|c|c|c|c|c|c|c|c|c|c|}
\hline $\begin{array}{l}\text { Field } \\
\text { No. }\end{array}$ & Lab. No. & Lat. N & Long. W & $\begin{array}{l}\text { Quadrangle } \\
(71 / 2 \mathrm{~min})\end{array}$ & County & Rock & $\begin{array}{l}\text { Scintill- } \\
\text { ometer } \\
\text { reading } \\
\text { in field } \\
\text { (c.p.s.) }\end{array}$ & $\underset{(p p m)}{U}$ & $\begin{array}{c}\text { Th } \\
\text { (ppm) }\end{array}$ & $\underset{(\mathrm{ppm})}{\mathrm{eU}}$ & Th/U \\
\hline $\begin{array}{l}784012 \\
784013 \\
784014 \\
784015\end{array}$ & $\begin{array}{l}\text { M138009 } \\
\text { M138010 } \\
\text { M138011 } \\
\text { M138012 }\end{array}$ & $\begin{array}{l}35^{\circ} 50^{\prime} 55^{\prime \prime} \\
36^{\circ} 03^{\prime} 20^{\prime \prime} \\
35^{\circ} 57^{\prime} 50^{\prime \prime} \\
36^{\circ} 08^{\prime} 20^{\prime \prime}\end{array}$ & $\begin{array}{l}121^{\circ} 02^{\prime} 05^{\prime \prime} \\
121^{\circ} 15^{\prime} 00^{\prime \prime} \\
120^{\circ} 38^{\prime} 35^{\prime \prime} \\
121^{\circ} 13^{\prime} 00^{\prime \prime}\end{array}$ & $\begin{array}{l}\text { Bryson } \\
\text { Bear Canyon } \\
\text { Valleton } \\
\text { Thompson Canyon }\end{array}$ & $\begin{array}{l}\mathbf{M} \\
\mathbf{M} \\
\mathbf{M} \\
\mathbf{M}\end{array}$ & $\begin{array}{l}\text { di } \\
\text { po } \\
\text { po } \\
\mathrm{mc}\end{array}$ & $\begin{array}{c}140 \\
200-220 \\
130-150 \\
140-150\end{array}$ & $\begin{array}{c}14.0 \\
21.8 \\
8.71 \\
10.5\end{array}$ & $\begin{array}{l}<6.7 \\
<6.5 \\
<5.1 \\
<4.4\end{array}$ & $\begin{array}{l}--- \\
--- \\
--\end{array}$ & $\begin{array}{l}<0.48 \\
<0.30 \\
<0.59 \\
<0.42\end{array}$ \\
\hline $\begin{array}{l}784016 \\
784017 \\
784018 \\
784019\end{array}$ & $\begin{array}{l}\text { M138013 } \\
\text { M138014 } \\
\text { M138015 } \\
\text { M138016 }\end{array}$ & $\begin{array}{l}36^{\circ} 08^{\prime} 30^{\prime \prime} \\
36^{\circ} 13^{\prime} 35^{\prime \prime} \\
36^{\circ} 13^{\prime} 40^{\prime \prime} \\
36^{\circ} 13^{\prime} 40^{\prime \prime}\end{array}$ & $\begin{array}{l}121^{\circ} 12^{\prime} 30^{\prime \prime} \\
121^{\circ} 16^{\prime} 40^{\prime \prime} \\
121^{\circ} 16^{\prime} 40^{\prime \prime} \\
121^{\circ} 16^{\prime} 40^{\prime \prime}\end{array}$ & $\begin{array}{l}\text { Thompson Canyon } \\
\text { Reliz Canyon } \\
\text { Reliz Canyon } \\
\text { Reliz Canyon }\end{array}$ & $\begin{array}{l}\mathbf{M} \\
\mathbf{M} \\
\mathbf{M} \\
\mathbf{M}\end{array}$ & $\begin{array}{l}\text { po } \\
\text { po } \\
\text { po } \\
\text { do }\end{array}$ & $\begin{array}{l}200-220 \\
110-130 \\
180-190 \\
120-130\end{array}$ & $\begin{array}{c}14.3 \\
4.13 \\
9.65 \\
2.28\end{array}$ & $\begin{array}{r}<5.3 \\
4.4 \\
<4.6 \\
<1.7\end{array}$ & $\begin{array}{l}--- \\
-- \\
--\end{array}$ & $\begin{array}{r}<0.37 \\
1.07 \\
<0.48 \\
<0.75\end{array}$ \\
\hline $\begin{array}{l}784020 \\
802001 \\
802002 \\
802003 \\
802004\end{array}$ & $\begin{array}{l}\text { M138017 } \\
\text { M144058 } \\
\text { M144059 } \\
\text { M144060 } \\
\text { M144061 }\end{array}$ & $\begin{array}{l}36^{\circ} 16^{\prime} 00^{\prime \prime} \\
36^{\circ} 14^{\prime} 10^{\prime \prime} \\
36^{\circ} 14^{\prime} 00^{\prime \prime} \\
36^{\circ} 14^{\prime} 30^{\prime \prime} \\
36^{\circ} 15^{\prime} 20^{\prime \prime}\end{array}$ & $\begin{array}{l}121^{\circ} 26^{\prime} 05^{\prime \prime} \\
121^{\circ} 28^{\prime} 15^{\prime \prime} \\
121^{\circ} 27^{\prime} 30^{\prime \prime} \\
121^{\circ} 26^{\prime} 00^{\prime \prime} \\
121^{\circ} 25^{\prime} 50^{\prime \prime}\end{array}$ & $\begin{array}{l}\text { Sycamore Flat } \\
\text { Junipero Serra Pk. } \\
\text { Junipero Serra Pk. } \\
\text { Junipero Serra Pk. } \\
\text { Sycamore Flat }\end{array}$ & $\begin{array}{l}\mathbf{M} \\
\mathbf{M} \\
\mathbf{M} \\
\mathbf{M} \\
\mathbf{M}\end{array}$ & $\begin{array}{l}\text { po } \\
\text { mc } \\
\text { mc } \\
\text { mc } \\
\text { po }\end{array}$ & $\begin{array}{l}250-270 \\
200-220 \\
120-140 \\
130-150 \\
170-190\end{array}$ & $\begin{array}{l}22.4 \\
12.3 \\
6.01 \\
7.00 \\
8.95\end{array}$ & $\begin{array}{l}<5.9 \\
16.9 \\
<6.3 \\
<6.5 \\
12 .\end{array}$ & $\begin{array}{r}--- \\
30 \\
20 \\
10 \\
40\end{array}$ & $\begin{array}{r}<0.26 \\
1.37 \\
<1.05 \\
<0.93 \\
1.34\end{array}$ \\
\hline $\begin{array}{l}802005 \\
802006 \\
802007 \\
802008 \\
802009\end{array}$ & $\begin{array}{l}\text { M144062 } \\
\text { M144063 } \\
\text { M144064 } \\
\text { M144065 } \\
\text { M144066 }\end{array}$ & $\begin{array}{l}36^{\circ} 16^{\prime} 45^{\prime \prime} \\
36^{\circ} 15^{\prime} 30^{\prime \prime} \\
36^{\circ} 15^{\prime} 30^{\prime \prime} \\
36^{\circ} 16^{\prime} 50^{\prime \prime} \\
36^{\circ} 12^{\prime} 10^{\prime \prime}\end{array}$ & $\begin{array}{l}121^{\circ} 27^{\prime} 00^{\prime \prime} \\
121^{\circ} 25^{\prime} 20^{\prime \prime} \\
121^{\circ} 25^{\prime} 20^{\prime \prime} \\
121^{\circ} 19^{\prime} 20^{\prime \prime} \\
121^{\circ} 16^{\prime} 30^{\prime \prime}\end{array}$ & $\begin{array}{l}\text { Sycamore Flat } \\
\text { Sycamore Flat } \\
\text { Sycamore Flat } \\
\text { Paraiso Springs } \\
\text { Reliz Canyon }\end{array}$ & $\begin{array}{l}\mathbf{M} \\
\mathbf{M} \\
\mathbf{M} \\
\mathbf{M} \\
\mathbf{M}\end{array}$ & $\begin{array}{l}\mathrm{mc} \\
\text { ph } \\
\text { po } \\
\text { mp } \\
\text { po }\end{array}$ & $\begin{array}{l}180-200 \\
210-240 \\
130-140 \\
160-180 \\
130-140\end{array}$ & $\begin{array}{c}13.5 \\
83.4 \\
5.34 \\
5.81 \\
8.06\end{array}$ & $\begin{array}{c}23 . \\
<25 . \\
8.7 \\
12 . \\
17 .\end{array}$ & $\begin{array}{l}30 \\
70 \\
10 \\
20 \\
20\end{array}$ & $\begin{array}{r}1.70 \\
<0.30 \\
1.63 \\
2.07 \\
2.11\end{array}$ \\
\hline $\begin{array}{l}802010 \\
802011 \\
802012 \\
802013 \\
802014\end{array}$ & $\begin{array}{l}\text { M144067 } \\
\text { M144068 } \\
\text { M144069 } \\
\text { M144070 } \\
\text { M144071 }\end{array}$ & $\begin{array}{l}36^{\circ} 13^{\prime} 50^{\prime \prime} \\
36^{\circ} 05^{\prime} 40^{\prime \prime} \\
36^{\circ} 04^{\prime} 40^{\prime \prime} \\
36^{\circ} 03^{\prime} 40^{\prime \prime} \\
36^{\circ} 02^{\prime} 30^{\prime \prime}\end{array}$ & $\begin{array}{l}121^{\circ} 16^{\prime} 50^{\prime \prime} \\
121^{\circ} 09^{\prime} 50^{\prime \prime} \\
121^{\circ} 10^{\prime} 50^{\prime \prime} \\
121^{\circ} 11^{\prime} 00^{\prime \prime} \\
121^{\circ} 13^{\prime} 20^{\prime \prime}\end{array}$ & $\begin{array}{l}\text { Reliz Canyon } \\
\text { Cosio Knob } \\
\text { Cosio Knob } \\
\text { Cosio Knob } \\
\text { Cosio Knob }\end{array}$ & $\begin{array}{l}\mathbf{M} \\
\mathbf{M} \\
\mathbf{M} \\
\mathbf{M} \\
\mathbf{M}\end{array}$ & $\begin{array}{l}\text { po } \\
\text { po } \\
\text { po } \\
\text { po } \\
\text { po }\end{array}$ & $\begin{array}{r}110-120 \\
90-100 \\
90-110 \\
90-100 \\
100-120\end{array}$ & $\begin{array}{l}8.56 \\
6.30 \\
6.65 \\
7.07 \\
2.48\end{array}$ & $\begin{array}{r}<9.2 \\
9.4 \\
<8.8 \\
<9.4 \\
7.0\end{array}$ & $\begin{array}{l}20 \\
10 \\
20 \\
10 \\
10\end{array}$ & $\begin{array}{r}<1.07 \\
1.49 \\
<1.32 \\
<1.33 \\
2.82\end{array}$ \\
\hline $\begin{array}{l}802015 \\
802016 \\
802017 \\
802018 \\
802019\end{array}$ & $\begin{array}{l}\text { M144072 } \\
\text { M144073 } \\
\text { M144074 } \\
\text { M144075 } \\
\text { M144076 }\end{array}$ & $\begin{array}{l}36^{\circ} 02^{\prime} 30^{\prime \prime} \\
36^{\circ} 04^{\prime} 45^{\prime \prime} \\
36^{\circ} 04^{\prime} 35^{\prime \prime} \\
36^{\circ} 04^{\prime} 40^{\prime \prime} \\
36^{\circ} 04^{\prime} 40^{\prime \prime}\end{array}$ & $\begin{array}{l}121^{\circ} 13^{\prime} 20^{\prime \prime} \\
121^{\circ} 15^{\prime} 10^{\prime \prime} \\
121^{\circ} 16^{\prime} 40^{\prime \prime} \\
121^{\circ} 16^{\prime} 35^{\prime \prime} \\
121^{\circ} 16^{\prime} 35^{\prime \prime}\end{array}$ & $\begin{array}{l}\text { Cosio Knob } \\
\text { Bear Canyon } \\
\text { Bear Canyon } \\
\text { Bear Canyon } \\
\text { Bear Canyon }\end{array}$ & $\begin{array}{l}\mathbf{M} \\
\mathbf{M} \\
\mathbf{M} \\
\mathbf{M} \\
\mathbf{M}\end{array}$ & $\begin{array}{l}\text { po } \\
\text { po } \\
\text { ss } \\
\text { ss } \\
\text { ch }\end{array}$ & $\begin{array}{l}140-150 \\
100-120 \\
100-110 \\
200-210 \\
110-120\end{array}$ & $\begin{array}{c}10.2 \\
5.04 \\
6.42 \\
45.0 \\
1.73\end{array}$ & $\begin{array}{l}18 . \\
<7.2 \\
<4.9 \\
<16 . \\
<3.1\end{array}$ & $\begin{array}{l}60 \\
10 \\
10 \\
60 \\
10\end{array}$ & $\begin{array}{l}1.77 \\
<1.43 \\
<0.76 \\
<0.36 \\
<1.79\end{array}$ \\
\hline $\begin{array}{l}802020 \\
802021 \\
802022 \\
802023 \\
802024\end{array}$ & $\begin{array}{l}\text { M144077 } \\
\text { M144078 } \\
\text { M144079 } \\
\text { M144080 } \\
\text { M144081 }\end{array}$ & $\begin{array}{l}35^{\circ} 53^{\prime} 50^{\prime \prime} \\
35^{\circ} 54^{\prime} 50^{\prime \prime} \\
35^{\circ} 52^{\prime} 00^{\prime \prime} \\
35^{\circ} 51^{\prime} 05^{\prime \prime} \\
35^{\circ} 46^{\prime} 40^{\prime \prime}\end{array}$ & $\begin{array}{l}121^{\circ} 06^{\prime} 00^{\prime \prime} \\
121^{\circ} 05^{\prime} 10^{\prime \prime} \\
121^{\circ} 03^{\prime} 40^{\prime \prime} \\
121^{\circ} 03^{\prime} 05^{\prime \prime} \\
120^{\circ} 53^{\prime} 20^{\prime \prime}\end{array}$ & $\begin{array}{l}\text { Williams Hill } \\
\text { Williams Hill } \\
\text { Bryson } \\
\text { Bryson } \\
\text { Tierra Redonda Mtn. }\end{array}$ & $\begin{array}{c}\mathbf{M} \\
\mathbf{M} \\
\mathbf{M} \\
\mathbf{M} \\
\text { SLO }\end{array}$ & $\begin{array}{l}\text { po } \\
\text { po } \\
\text { po } \\
\mathrm{mc} \\
\mathrm{mc}\end{array}$ & $\begin{array}{l}110-120 \\
100-110 \\
120-140 \\
130-150 \\
100-120\end{array}$ & $\begin{array}{l}9.93 \\
12.2 \\
11.3 \\
19.9 \\
10.5\end{array}$ & $\begin{array}{l}<10 . \\
<11 \\
<8.7 \\
<17 . \\
<9.6\end{array}$ & $\begin{array}{l}40 \\
30 \\
40 \\
10 \\
40\end{array}$ & $\begin{array}{l}<1.01 \\
<0.90 \\
<0.77 \\
<0.85 \\
<0.91\end{array}$ \\
\hline $\begin{array}{l}802025 \\
802026 \\
802027 \\
802028 \\
802029\end{array}$ & $\begin{array}{l}\text { M144082 } \\
\text { M144083 } \\
\text { M144084 } \\
\text { M144085 } \\
\text { M144086 }\end{array}$ & $\begin{array}{l}35^{\circ} 47^{\prime} 40^{\prime \prime} \\
35^{\circ} 47^{\prime} 35^{\prime \prime} \\
35^{\circ} 54^{\prime} 00^{\prime \prime} \\
36^{\circ} 06^{\prime} 40^{\prime \prime} \\
36^{\circ} 00^{\prime} 10^{\prime \prime}\end{array}$ & $\begin{array}{l}120^{\circ} 53^{\prime} 00^{\prime \prime} \\
120^{\circ} 51^{\prime} 50^{\prime \prime} \\
120^{\circ} 57^{\prime} 30^{\prime \prime} \\
121^{\circ} 07^{\prime} 20^{\prime \prime} \\
121^{\circ} 04^{\prime} 00^{\prime \prime}\end{array}$ & $\begin{array}{l}\text { Tierra Redonda Mtn. } \\
\text { Bradley } \\
\text { Hames Valley } \\
\text { Espinosa Canyon } \\
\text { Espinosa Canyon }\end{array}$ & $\begin{array}{l}\mathbf{M} \\
\mathbf{M} \\
\mathbf{M} \\
\mathbf{M} \\
\mathbf{M}\end{array}$ & $\begin{array}{l}\text { po } \\
\text { ch } \\
\text { po } \\
\text { po } \\
\text { pc }\end{array}$ & $\begin{array}{r}140-160 \\
90-110 \\
90-100 \\
90-100 \\
80\end{array}$ & $\begin{array}{l}6.02 \\
6.17 \\
6.89 \\
5.88 \\
2.40\end{array}$ & $\begin{array}{r}<6.6 \\
7.9 \\
<6.7 \\
<9.1 \\
<4.9\end{array}$ & $\begin{array}{l}10 \\
10 \\
10 \\
30 \\
10\end{array}$ & $\begin{array}{r}<1.10 \\
1.28 \\
<0.97 \\
<1.55 \\
<2.04\end{array}$ \\
\hline $\begin{array}{l}802030 \\
802031 \\
802032 \\
802033 \\
802034\end{array}$ & $\begin{array}{l}\text { M144087 } \\
\text { M144088 } \\
\text { M144089 } \\
\text { M144090 } \\
\text { M144091 }\end{array}$ & $\begin{array}{l}35^{\circ} 58^{\prime} 30^{\prime \prime} \\
35^{\circ} 59^{\prime} 10^{\prime \prime} \\
35^{\circ} 59^{\prime} 15^{\prime \prime} \\
35^{\circ} 45^{\prime} 45^{\prime \prime} \\
35^{\circ} 56^{\prime} 45^{\prime \prime}\end{array}$ & $\begin{array}{l}121^{\circ} 03^{\prime} 00^{\prime \prime} \\
121^{\circ} 01^{\prime} 10^{\prime \prime} \\
120^{\circ} 57^{\prime} 40^{\prime \prime} \\
120^{\circ} 53^{\prime} 00^{\prime \prime} \\
120^{\circ} 39^{\prime} 45^{\prime \prime}\end{array}$ & $\begin{array}{l}\text { Williams Hill } \\
\text { Williams Hill } \\
\text { Hames Valley } \\
\text { Tierra Redonda Mtn. } \\
\text { Valleton }\end{array}$ & $\begin{array}{c}\mathbf{M} \\
\mathbf{M} \\
\mathbf{M} \\
\text { SLO } \\
\mathbf{M}\end{array}$ & $\begin{array}{l}\text { po } \\
\mathrm{mp} \\
\mathrm{mp} \\
\mathrm{mc} \\
\mathrm{mp}\end{array}$ & $\begin{array}{r}100-110 \\
160-170 \\
130-140 \\
90-100 \\
140-160\end{array}$ & $\begin{array}{c}6.80 \\
10.9 \\
2.84 \\
6.28 \\
15.3\end{array}$ & $\begin{array}{l}<6.4 \\
16 . \\
12.3 \\
<8.2 \\
<12\end{array}$ & $\begin{array}{l}10 \\
20 \\
10 \\
10 \\
10\end{array}$ & $\begin{array}{r}<0.94 \\
1.47 \\
4.33 \\
<1.31 \\
<0.78\end{array}$ \\
\hline $\begin{array}{l}802035 \\
802036 \\
802037 \\
802038 \\
802039\end{array}$ & $\begin{array}{l}\text { M144092 } \\
\text { M144093 } \\
\text { M144094 } \\
\text { M144095 } \\
\text { M144096 }\end{array}$ & $\begin{array}{l}35^{\circ} 55^{\prime} 50^{\prime \prime} \\
35^{\circ} 59^{\prime} 40^{\prime \prime} \\
35^{\circ} 39^{\prime} 30^{\prime \prime} \\
35^{\circ} 38^{\prime} 65^{\prime \prime} \\
35^{\circ} 36^{\prime} 20^{\prime \prime}\end{array}$ & $\begin{array}{l}120^{\circ} 40^{\prime} 40^{\prime \prime} \\
120^{\circ} 38^{\prime} 45^{\prime \prime} \\
120^{\circ} 46^{\prime} 30^{\prime \prime} \\
120^{\circ} 51^{\prime} 20^{\prime \prime} \\
120^{\circ} 50^{\prime} 20^{\prime \prime}\end{array}$ & $\begin{array}{l}\text { Valleton } \\
\text { Valleton } \\
\text { Adelaida } \\
\text { Adelaida } \\
\text { York Mountain }\end{array}$ & $\begin{array}{l}\text { M } \\
\mathbf{M} \\
\text { SLO } \\
\text { SLO } \\
\text { SLO }\end{array}$ & $\begin{array}{l}\text { di } \\
\text { po } \\
\mathrm{mc} \\
\mathrm{mc} \\
\mathrm{mc}\end{array}$ & $\begin{array}{c}60-65 \\
150-170 \\
250-270 \\
120-130 \\
150-160\end{array}$ & $\begin{array}{c}2.66 \\
12.7 \\
52.6 \\
6.74 \\
16.3\end{array}$ & $\begin{array}{l}7.4 \\
13 . \\
<19 . \\
<8.2 \\
<12 .\end{array}$ & $\begin{array}{l}30 \\
20 \\
60 \\
10 \\
30\end{array}$ & $\begin{array}{r}2.78 \\
1.02 \\
<0.36 \\
<1.22 \\
<0.74\end{array}$ \\
\hline $\begin{array}{l}802040 \\
802041 \\
802042 \\
802043 \\
802044\end{array}$ & $\begin{array}{l}\text { M144097 } \\
\text { M144098 } \\
\text { M144099 } \\
\text { M144100 } \\
\text { M144101 }\end{array}$ & $\begin{array}{l}35^{\circ} 37^{\prime} 35^{\prime \prime} \\
35^{\circ} 33^{\prime} 40^{\prime \prime} \\
35^{\circ} 28^{\prime} 50^{\prime \prime} \\
35^{\circ} 28^{\prime} 45^{\prime \prime} \\
35^{\circ} 28^{\prime} 45^{\prime \prime}\end{array}$ & $\begin{array}{l}120^{\circ} 44^{\prime} 00^{\prime \prime} \\
120^{\circ} 39^{\prime} 00^{\prime \prime} \\
120^{\circ} 24^{\prime} 45^{\prime \prime} \\
120^{\circ} 24^{\prime} 50^{\prime \prime} \\
120^{\circ} 24^{\prime} 50^{\prime \prime}\end{array}$ & $\begin{array}{l}\text { Paso Robles } \\
\text { Templeton } \\
\text { Wilson Corner } \\
\text { Wilson Corner } \\
\text { Wilson Corner }\end{array}$ & $\begin{array}{l}\text { SLO } \\
\text { SLO } \\
\text { SLO } \\
\text { SLO } \\
\text { SLO }\end{array}$ & $\begin{array}{l}\text { po } \\
\text { po } \\
\text { tu } \\
\text { ch } \\
\text { ms }\end{array}$ & $\begin{array}{c}60-70 \\
120-125 \\
210-240 \\
95-110 \\
190-210\end{array}$ & $\begin{array}{c}4.72 \\
22.4 \\
9.45 \\
2.31 \\
9.88\end{array}$ & $\begin{array}{c}<7.2 \\
<14 . \\
29.2 \\
<3.4 \\
33.0\end{array}$ & $\begin{array}{l}10 \\
10 \\
50 \\
10 \\
30\end{array}$ & $\begin{array}{r}<1.53 \\
<0.63 \\
3.09 \\
<1.47 \\
3.34\end{array}$ \\
\hline $\begin{array}{l}802045 \\
802046 \\
802047 \\
802048 \\
802049 \\
802050\end{array}$ & $\begin{array}{l}\text { M144102 } \\
\text { M144103 } \\
\text { M144104 } \\
\text { M144105 } \\
\text { M144106 } \\
\text { M144107 }\end{array}$ & $\begin{array}{l}35^{\circ} 28^{\prime} 45^{\prime \prime} \\
35^{\circ} 28^{\prime} 35^{\prime \prime} \\
35^{\circ} 54^{\prime} 30^{\prime \prime} \\
35^{\circ} 14^{\prime} 15^{\prime \prime} \\
35^{\circ} 41^{\prime} 20^{\prime \prime} \\
35^{\circ} 53^{\prime} 10^{\prime \prime}\end{array}$ & $\begin{array}{l}120^{\circ} 24^{\prime} 50^{\prime \prime} \\
120^{\circ} 24^{\prime} 40^{\prime \prime} \\
120^{\circ} 32^{\prime} 50^{\prime \prime} \\
120^{\circ} 46^{\prime} 40^{\prime \prime} \\
120^{\circ} 46^{\prime} 50^{\prime \prime} \\
121^{\circ} 03^{\prime} 55^{\prime \prime}\end{array}$ & $\begin{array}{l}\text { Wilson Corner } \\
\text { Wilson Corner } \\
\text { Stockdale Mountain } \\
\text { Adelaida } \\
\text { Adelaida } \\
\text { Williams Hill }\end{array}$ & $\begin{array}{l}\text { SLO } \\
\text { SLO } \\
\mathbf{M} \\
\text { SLO } \\
\text { SLO } \\
\text { M }\end{array}$ & $\begin{array}{l}\mathrm{ms} \\
\mathrm{mc} \\
\mathrm{mp} \\
\mathrm{mc} \\
\text { po } \\
\text { do }\end{array}$ & $\begin{array}{c}140-145 \\
100-110 \\
120-130 \\
65-70 \\
95-105 \\
60-65\end{array}$ & $\begin{array}{l}5.29 \\
4.52 \\
3.24 \\
2.80 \\
5.39 \\
6.44\end{array}$ & $\begin{array}{l}<9.1 \\
<4.8 \\
12.4 \\
<3.2 \\
<5.4 \\
<7.3\end{array}$ & $\begin{array}{l}20 \\
10 \\
10 \\
30 \\
10 \\
10\end{array}$ & $\begin{array}{r}<1.72 \\
<1.06 \\
3.83 \\
<1.14 \\
<1.00 \\
<1.13\end{array}$ \\
\hline
\end{tabular}


Table 2. Summary of uranium analyses of outcrop samples of Monterey Formation

\begin{tabular}{|c|c|c|c|c|c|}
\hline \multirow{2}{*}{\multicolumn{2}{|c|}{ Samples }} & \multirow{2}{*}{$\begin{array}{c}\text { Number of } \\
\text { samples }\end{array}$} & \multicolumn{3}{|c|}{ Uranium content (ppm) } \\
\hline & & & Range & Average & Median \\
\hline \multirow[b]{2}{*}{ Outcrop samples (fig. 2) } & A. All samples & 111 & $1.73-1854.73$ & 54.12 & 10.4 \\
\hline & $B$. Samples not from uranium prospects & 91 & $1.73-\quad 73.4$ & 13.35 & 8.40 \\
\hline \multirow{3}{*}{ Samples from Temblor Range (fig. 3) } & $A$. All samples & 39 & $2.58-1854.73$ & 132.31 & 33.6 \\
\hline & B. Samples from uranium prospects & 20 & $18.6-1854.73$ & 239.61 & 97.8 \\
\hline & C. Samples not from uranium prospects & 19 & $2.58-\quad 77.9$ & 19.36 & 12.2 \\
\hline \multirow[b]{2}{*}{$\begin{array}{l}\text { Samples from outside Temblor } \\
\text { Range (fig. 4). }\end{array}$} & $A$. All samples & 72 & $1.73-83.4$ & 10.6 & 7.36 \\
\hline & $\begin{array}{l}B \text {. Samples collected without respect to } \\
\text { radioactivity }\end{array}$ & 50 & $1.73-83.4$ & 11.1 & 6.77 \\
\hline \multirow{4}{*}{$\begin{array}{l}\text { Samples of porcelanite, porcelaneous } \\
\text { mudstone, and cherty porcelanite } \\
\text { (fig. 5). }\end{array}$} & s $A$. All samples & 64 & $2.40-767$ & 48.41 & 10.8 \\
\hline & $B$. Samples not from uranium prospects & 49 & $2.40-55.4$ & 11.42 & 8.56 \\
\hline & C. Samples from outside Temblor Range & 40 & $2.40-55.4$ & 10.46 & 8.31 \\
\hline & $A$. All sa & 21 & $2.26-1854.73$ & 119.92 & 14.0 \\
\hline Samples of diatomite and & $B$. Samples not from uranium prospects & 15 & $2.26-77.9$ & 16.36 & 8.40 \\
\hline diatomaceous mudstone (fig. 6). & C. Samples from outside Temblor Range & 6 & $2.26-\quad 14.0$ & 6.43 & 5.62 \\
\hline \multicolumn{2}{|l|}{ Calcareous mudstone (fig. 7) } & 13 & $2.80-52.6$ & 13.0 & 10.5 \\
\hline
\end{tabular}

biased by inclusion of samples from uranium prospects in the Temblor Range. If samples from prospects are omitted, however, the range of uranium content of the remaining samples was $2.40-55.4 \mathrm{ppm}$, the average was $11.42 \mathrm{ppm}$, and the median was $8.56 \mathrm{ppm}$. Samples of Monterey porcelanite from outside the Temblor Range had uranium content of $2.40-55.4 \mathrm{ppm}$, averaged $10.46 \mathrm{ppm}$, and had a median of $8.31 \mathrm{ppm}$-values not appreciably different from those obtained for Monterey porcelanite samples including those from the Temblor Range, but outside uranium prospects. This suggests that, except for the prospects, the uranium content of porcelanite in the Temblor Range is about the same as that elsewhere.

The uranium content of 21 samples of diatomite from the Monterey Formation (fig. 6) ranged from about 2 to $1854 \mathrm{ppm}$, had an average value of $119.92 \mathrm{ppm}$, and had a median value of $14.0 \mathrm{ppm}$. Again, these values are biased by inclusion of diatomite samples from uranium prospects. Only six samples of diatomite from outside the Temblor Range were analyzed-too few from which to draw any well-founded conclusions-but the uranium content of these six ranged from 2.26 to $14.0 \mathrm{ppm}$, had an average value of $6.43 \mathrm{ppm}$, and had a median value of $5.26 \mathrm{ppm}$, values which are somewhat greater than for many kinds of rock.

The uranium content of 13 samples of calcareous mudstone from the Monterey Formation in the southern Salinas Valley area (fig. 7) ranged from 2.80 to $52.6 \mathrm{ppm}$, had an average value of $13.0 \mathrm{ppm}$, and had a median value of $10.5 \mathrm{ppm}$. The uranium content of four samples of similar rock from Newport Bay (not included in table 1 or fig. 7) ranged from 5.33 to $11.6 \mathrm{ppm}$ and had an average value of $8.01 \mathrm{ppm}$. These values for Monterey 

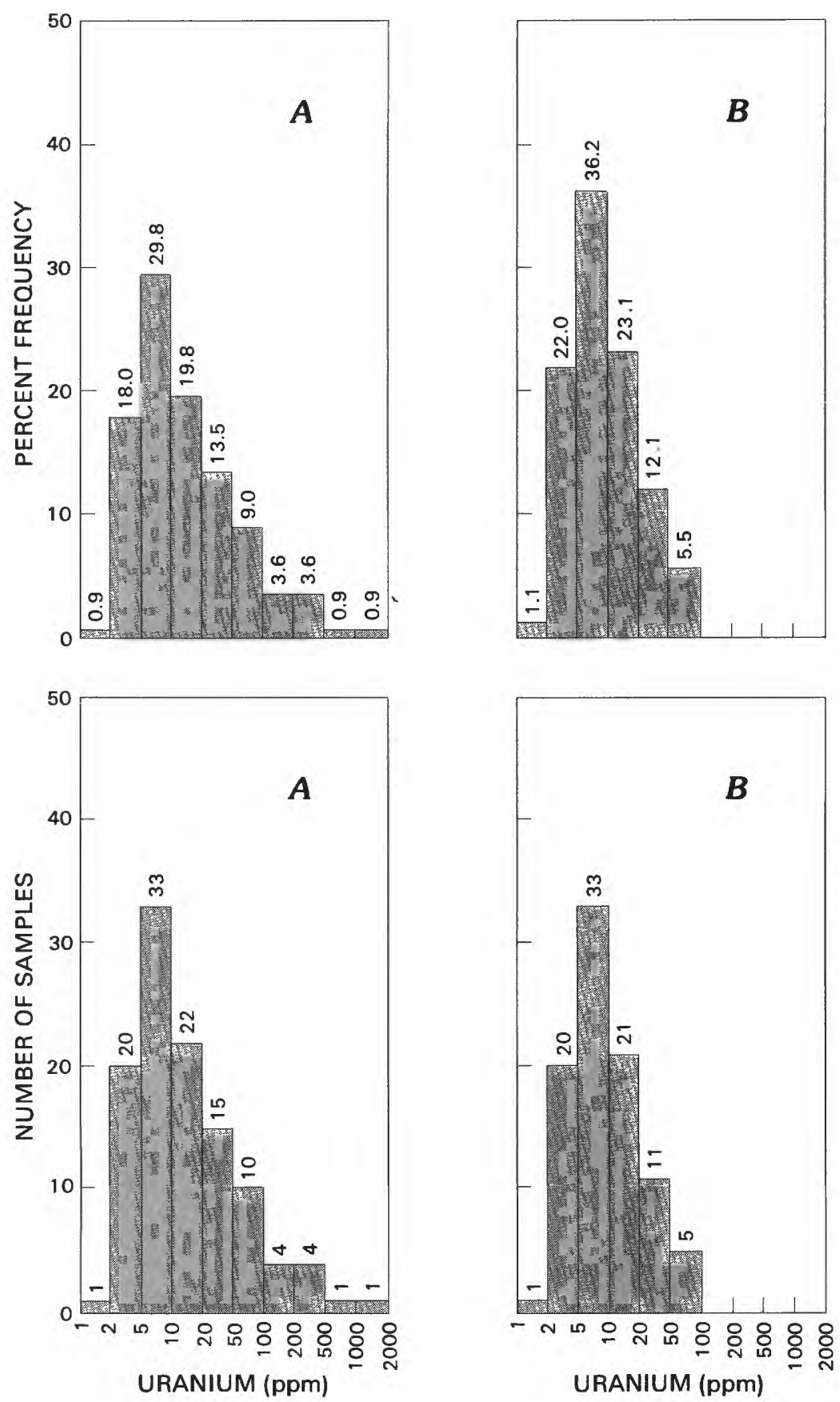

Figure 2. Histograms showing uranium content of outcrop samples; values are listed in table 2. A, All samples. B, Samples not from uranium prospects. 

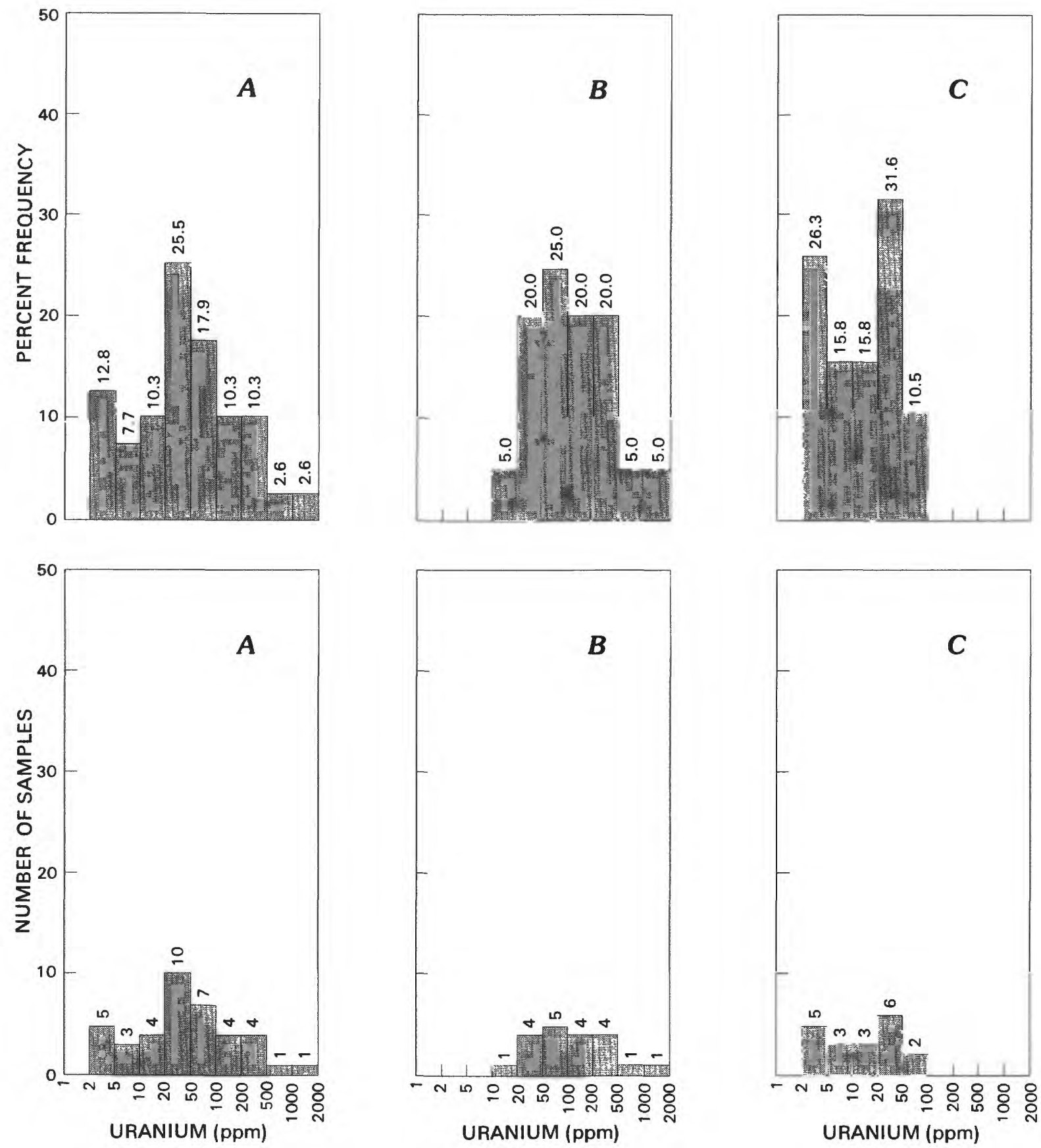

Figure 3. Histograms showing uranium content of outcrop samples from Temblor Range; values are listed in table 2. A, All samples. B, Samples from uranium prospects. C, Samples not from uranium prospects. 

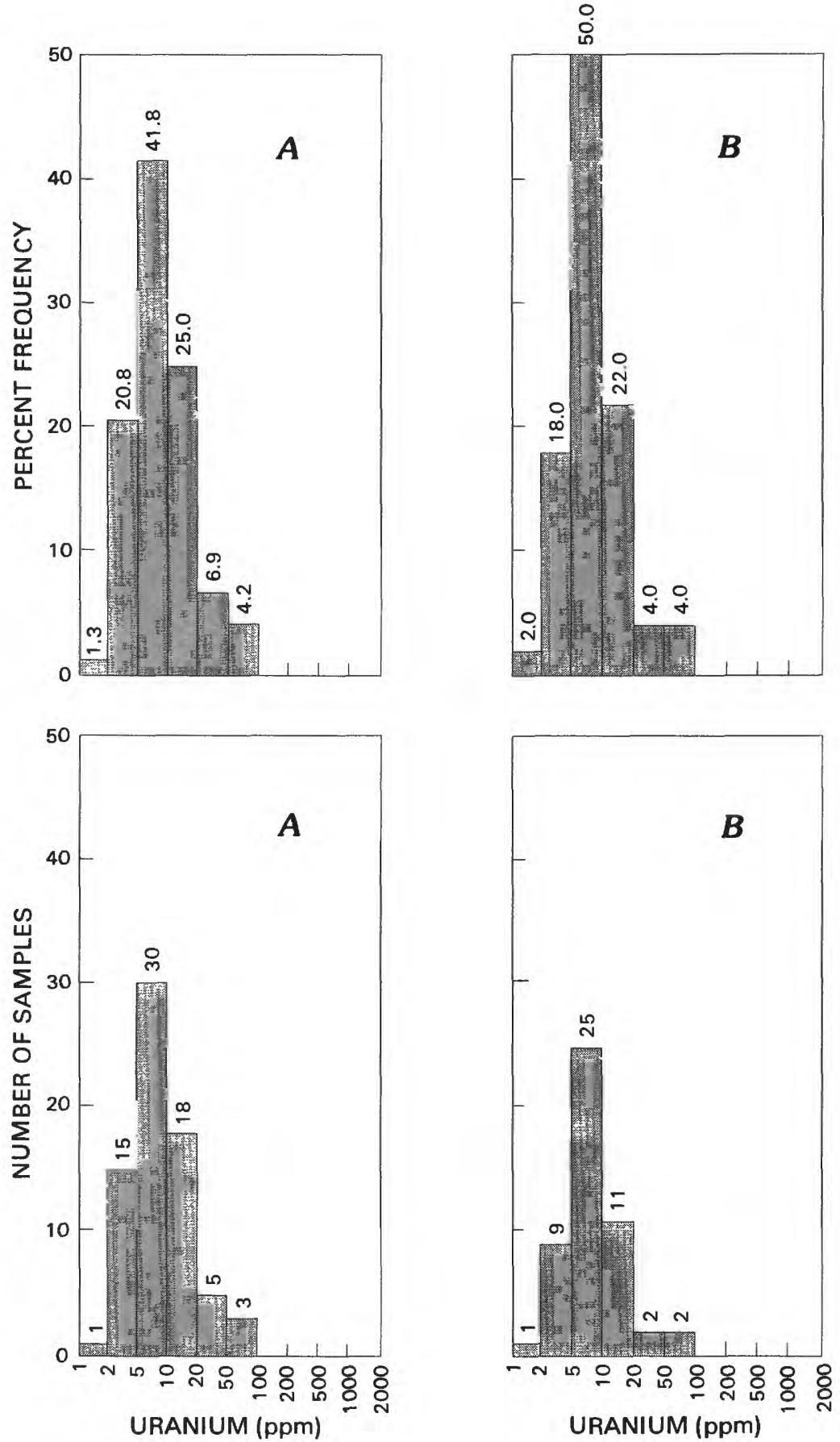

Figure 4. Histograms showing uranium content of outcrop samples from outside Temblor Range; values are listed in table 2. A, All samples. B, Samples collected without respect to radioactivity. 

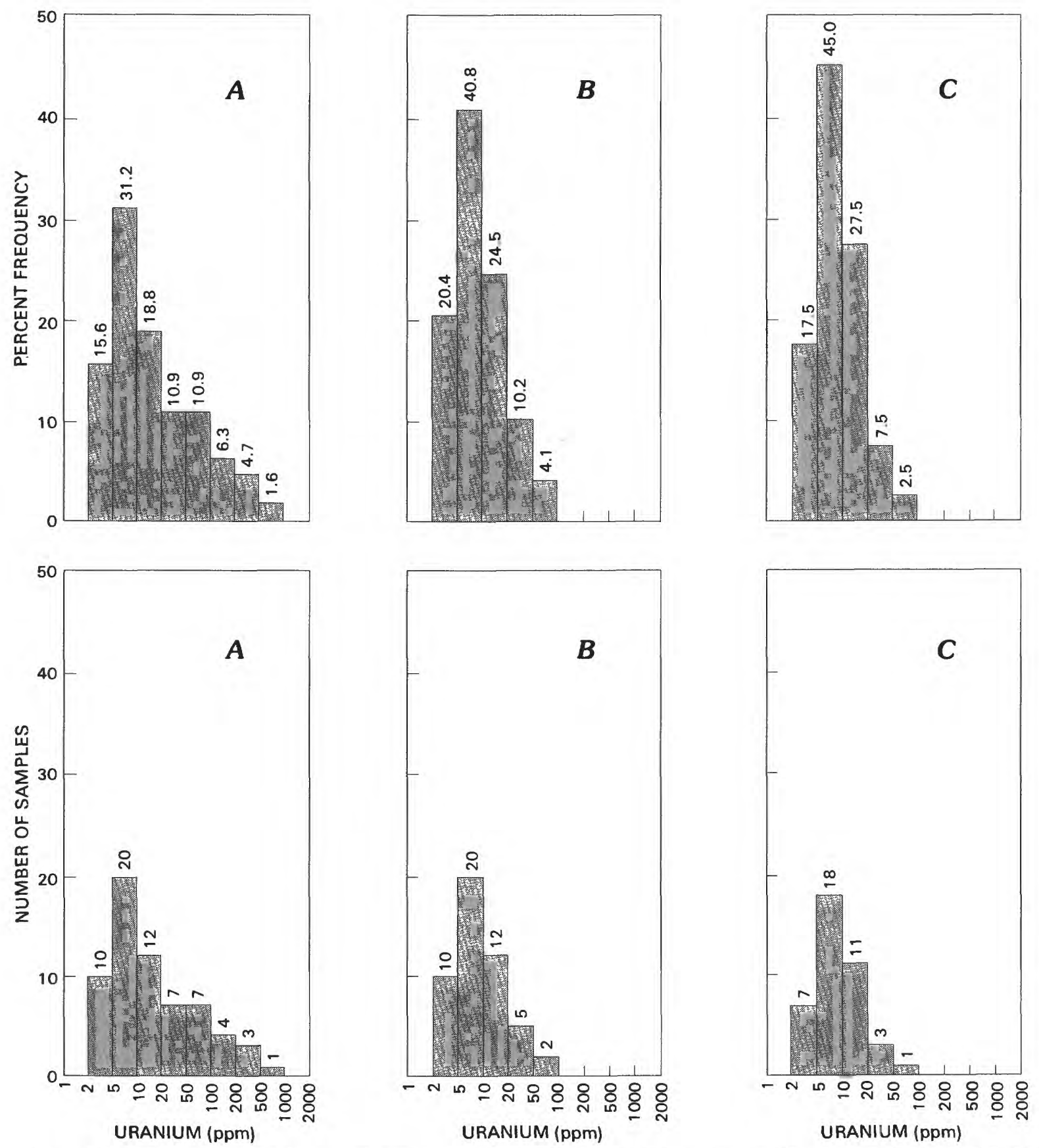

Figure 5. Histograms showing uranium content of porcelanite, porcelaneous mudstone, and cherty porcelanite; values are listed in table 2 . A, All samples. $B$, Samples not from uranium prospects. $C$, Samples from outside the Temblor Range. 

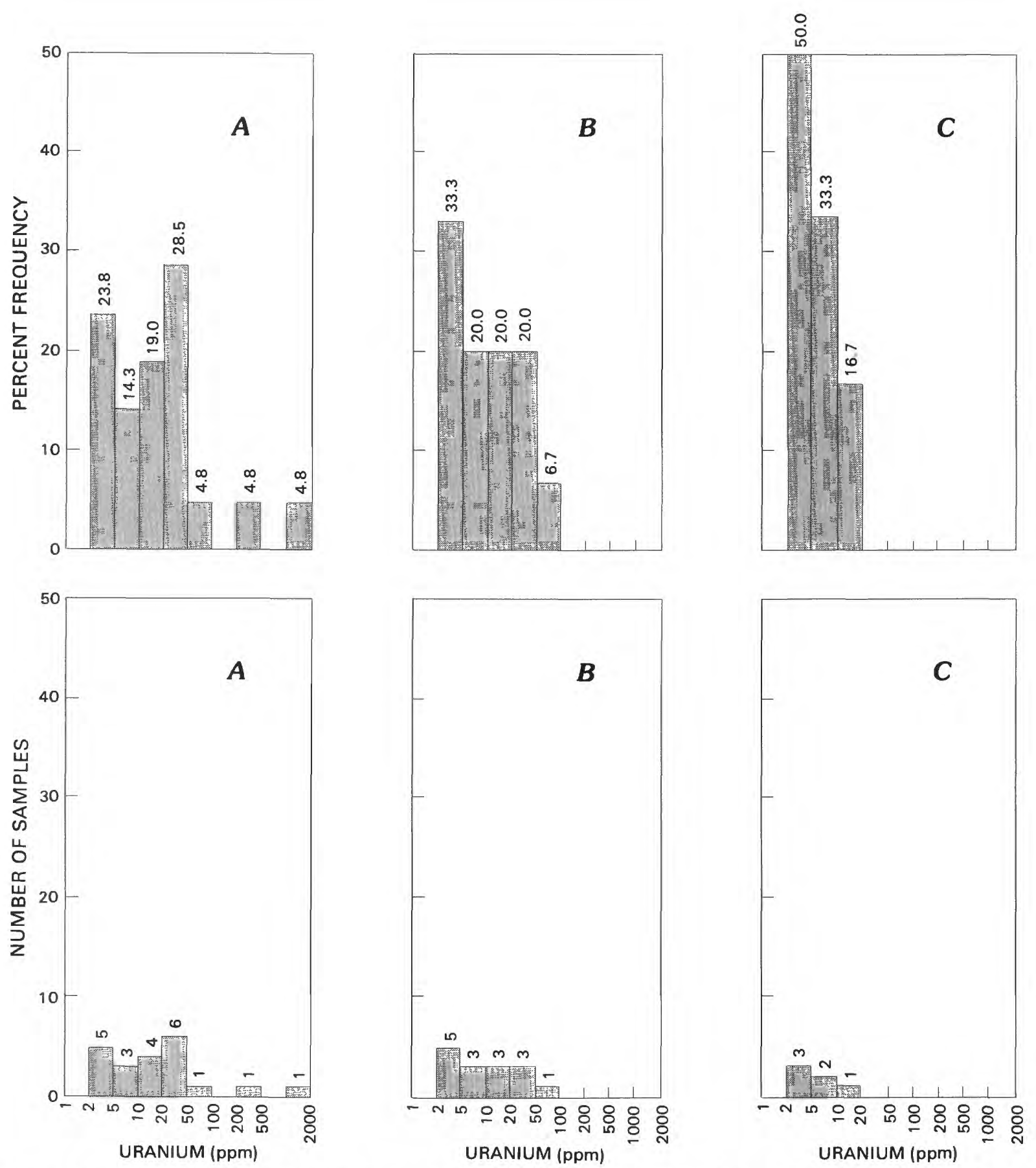

Figure 6. Histograms showing uranium content of samples of diatomite and diatomaceous mudstone; values are listed in table 2. A, All samples. B, Samples not from uranium prospects. C, Samples from outside the Temblor Range. 

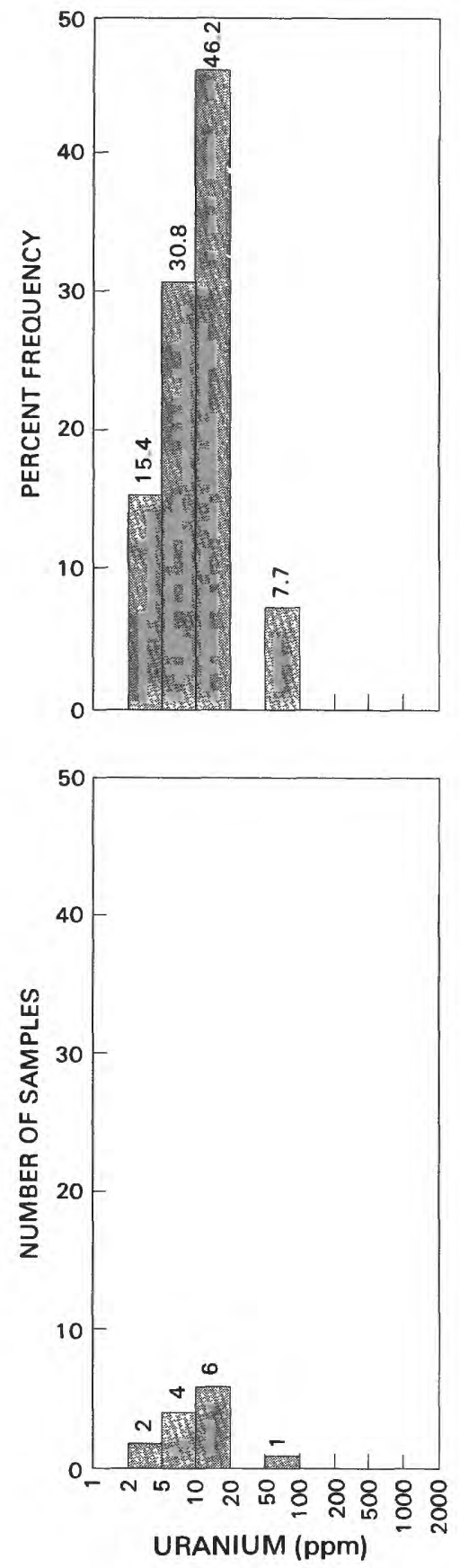

Figure 7. Histograms showing uranium content of samples of calcareous mudstone; values are listed in table 2.

calcareous mudstone are close to those for Monterey porcelanite from outside uranium prospects.

Only a few analyses of other kinds of rock from the Monterey Formation were made, all samples from outside the Temblor Range. The uranium content of four samples of chert ranged from 1.73 to $6.17 \mathrm{ppm}$, had an average value of $3.36 \mathrm{ppm}$, and had a median value of $2.76 \mathrm{ppm}$. Two samples of dolomite contained 2.28 and
$6.44 \mathrm{ppm}$ uranium, two samples of bedded pellet phosphorite contained 39.8 and $83.4 \mathrm{ppm}$ uranium, and a sample of vitric tuff contained $9.45 \mathrm{ppm}$ uranium. Chert seems generally to have the lowest uranium content of the major kinds of rock in the Monterey. Dolomite beds and concretions also appear to have a uranium content lower than for most rocks in the formation. The high values for phosphorite are to be expected, considering the normal affinity of uranium for phosphorus. The uranium content of the tuff is in the normal range for siliceous volcanic rocks.

Dependable values for thorium content were obtained for only 26 outcrop samples from the Monterey Formation (table 3). If samples of tuff and siliceous mudstone are omitted, the thorium content of the remaining samples-more typical of the Monterey-ranged from 4.4 to $18 \mathrm{ppm}$, had an average value of $11.24 \mathrm{ppm}$, and had a median value of $10.45 \mathrm{ppm}$. The thorium-uranium ratio ranges from 0.96 to 4.33 but more than half of the values are less than 2 , and nearly all are less than 3 (fig. 8)-ratio values that are less than for most rocks. Nearly all samples with uranium content greater than $3.5 \mathrm{ppm}$ have thorium-uranium ratios between 1 and 2 . There appears to be no other relation between either uranium or thorium values and the thorium-uranium ratio.

Ten outcrop samples from the Monterey Formation were analyzed by emission spectroscopy for 19 elements other than uranium and thorium (table 4). The samples had a wide range of uranium content, but they all showed no obvious relation between uranium and any of the other elements listed.

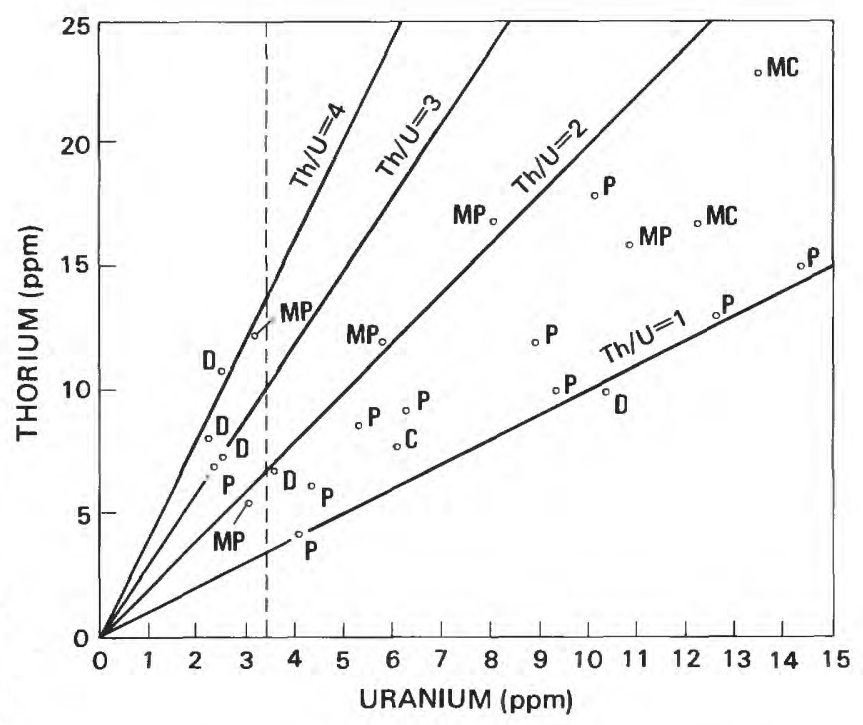

Figure 8. Graph showing thorium-uranium ratios for samples from Monterey Formation. Abbreviations for rocks: C, chert; $\mathrm{D}$, diatomite; MC, calcareous mudstone; MP, porcelaneous mudstone; $\mathrm{P}$, porcelanite. 
Table 3. Thorium analyses of outcrop samples from Monterey Formation [Abbreviations for rocks: ch, chert; di, diatomite; mc, calcareous mudstone; mp, porcelaneous mudstone; ms, siliceous mudstone; po, porcelanite; tu, tuff]

\begin{tabular}{lllccc}
\hline Field No. & Lab. No. & Rock & $\begin{array}{c}\text { Th } \\
(\mathrm{ppm})\end{array}$ & $\begin{array}{c}\mathrm{U} \\
(\mathrm{ppm})\end{array}$ & Th/U \\
\hline 764003 & D187773 & po & 6.02 & 4.37 & 1.38 \\
771001 & M131628 & di & 10. & 10.4 & 0.96 \\
771040 & M131634 & mp & 5.5 & 3.10 & 1.77 \\
771080 & M131638 & po & 10. & 9.34 & 1.07 \\
772009 & M131642 & po & 15. & 14.3 & 1.05 \\
& & & & & \\
772083 & M131649 & di & 10.9 & 2.58 & 4.22 \\
784004 & M138001 & di & 8.2 & 2.26 & 3.63 \\
784007 & M138004 & di & 6.8 & 3.58 & 1.90 \\
784017 & M138014 & po & 4.4 & 4.13 & 1.07 \\
802001 & M144058 & mc & 16.9 & 12.3 & 1.37 \\
& & & & & \\
802004 & M144061 & po & 12. & 8.95 & 1.34 \\
802005 & M144062 & mc & 23. & 13.5 & 1.70 \\
802007 & M144064 & po & 8.7 & 5.34 & 1.63 \\
802008 & M144065 & mp & 12. & 5.81 & 2.07 \\
802009 & M144066 & po & 17. & 8.06 & 2.11 \\
& & & & & \\
802011 & M144068 & po & 9.4 & 6.30 & 1.49 \\
802014 & M144071 & po & 7.0 & 2.48 & 2.82 \\
802015 & M144072 & po & 18. & 10.2 & 1.77 \\
802026 & M144083 & ch & 7.9 & 6.17 & 1.28 \\
802031 & M144088 & mp & 16. & 10.9 & 1.47 \\
& & & & & \\
802032 & M144089 & mp & 12.3 & 2.84 & 4.33 \\
802035 & M144092 & di & 7.4 & 2.66 & 2.78 \\
802036 & M144093 & po & 13. & 12.7 & 1.02 \\
802042 & M144099 & tu & 29.2 & 9.45 & 3.09 \\
802044 & M144101 & ms & 33.0 & 9.88 & 3.34 \\
802047 & M144104 & mp & 12.4 & 3.24 & 3.83 \\
\hline & & & & & \\
\hline
\end{tabular}

Table 4. Analyses by emission spectroscopy for 19 elements in samples of Monterey Formation that have a wide range of uranium content

[Abbreviations for rocks: di, diatomite; mp; porcelaneous mudstone; po, porcelanite]

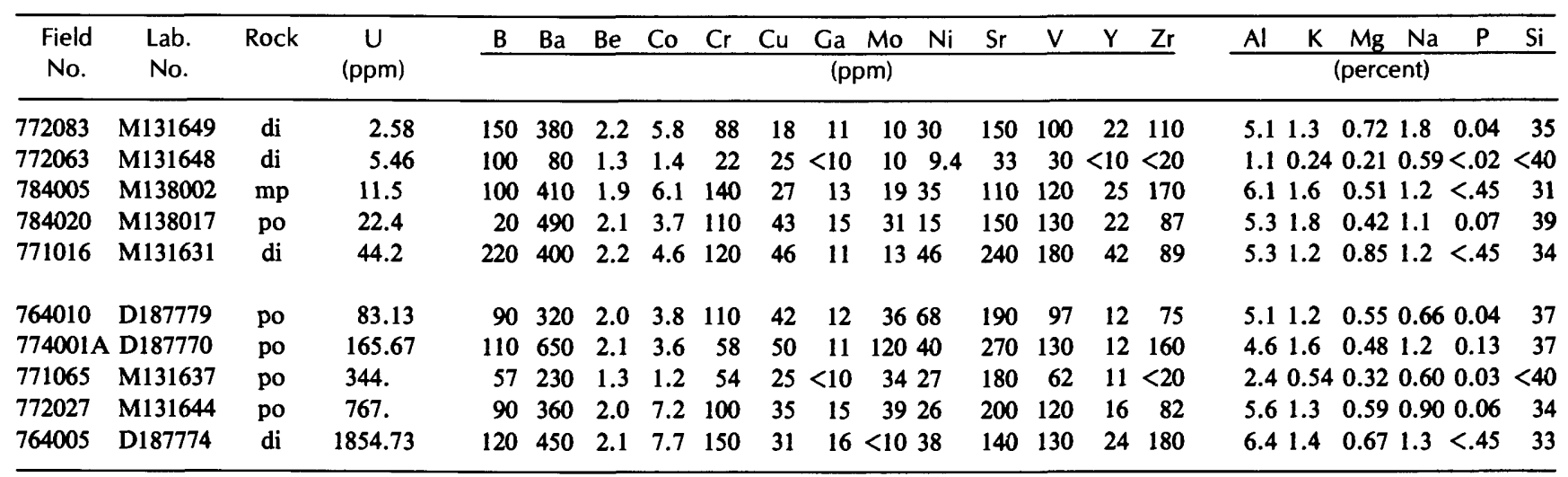


Table 5. Uranium and thorium content of core samples from the Monterey Formation near the Temblor Range

[Samples from wells of the Standard Oil Company of California located just east of Temblor Range in sec. 26, T. 12 N., R. 24 W.; Samples provided by L. A. Beyer and C. M. Isaacs. Abbreviations for rocks; md, diatomaceous mudstone; ms, siliceous mudstone; po, porcelanite; sl, siltstone. ---, leaders indicate not present]

\begin{tabular}{lccccccc}
\hline Well & Lab. No. & $\begin{array}{c}\text { Depth } \\
\text { (feet) }\end{array}$ & Rock & $\begin{array}{c}\mathrm{U} \\
(\mathrm{ppm})\end{array}$ & $\begin{array}{c}\text { Th } \\
(\mathrm{ppm})\end{array}$ & $\begin{array}{c}\mathrm{eU} \\
(\mathrm{ppm})\end{array}$ & Th/U \\
\hline Anza Pacific Corp. 59 & D192869 & 1265 & $\mathrm{md}$ & 18.87 & -- & 20 & --- \\
& D192870 & 1430 & po & 9.36 & -- & 20 & --- \\
& D192871 & 1489 & $\mathrm{~ms}$ & 10.52 & 21.00 & 30 & 2.00 \\
& D192872 & 1535 & po & 11.08 & -- & 10 & -- \\
& D192873 & 2077 & po & 8.20 & -- & 10 & -- \\
& & & & & & & \\
Ethel D-101 & D192874 & 1112 & $\mathrm{~ms}$ & 8.74 & -- & 10 & -- \\
& D192875 & 1165 & $\mathrm{~ms}$ & 6.58 & 10.09 & 20 & 1.53 \\
& $\mathrm{D} 192876$ & 1464 & $\mathrm{sl}$ & 8.17 & 18.26 & 20 & 2.24 \\
\hline
\end{tabular}

Table 6. Uranium, thorium, and organic carbon content of core samples from the Monterey Formation and related rocks from near Santa Maria

[Samples from wells of Union Oil Company; samples and stratigraphic data provided by C. M. Isaacs]

\begin{tabular}{|c|c|c|c|c|c|c|c|}
\hline Well & Lab. No. & $\begin{array}{l}\text { Depth } \\
\text { (feet) }\end{array}$ & $\underset{(p p m)}{U}$ & $\begin{array}{l}\text { Th } \\
\text { (ppm) }\end{array}$ & Th/U & $\begin{array}{l}\text { Organic carbon } \\
\text { (percent) }\end{array}$ & Remarks \\
\hline $\begin{array}{l}\text { Bradley } 1 \\
\text { (sec. 26, T. } 10 \text { N., } \\
\text { R. } 34 \text { W.) }\end{array}$ & $\begin{array}{l}\text { D236001 } \\
\text { D236000 }\end{array}$ & $\begin{array}{l}4156-4165 \\
4840-4849\end{array}$ & $\begin{array}{l}8.43 \\
13.4\end{array}$ & $\begin{array}{l}<4.7 \\
<6.2\end{array}$ & $\begin{array}{l}<0.56 \\
<0.46\end{array}$ & $\begin{array}{l}3.44 \\
14.4\end{array}$ & $\begin{array}{l}\text { Monterey Formation } \\
\text { Monterey Formation }\end{array}$ \\
\hline $\begin{array}{l}\text { Dome } 18 \\
\text { (sec. } 24, \text { T. } 9 \text { N., } \\
\text { R. } 34 \text { W.) }\end{array}$ & $\begin{array}{l}\text { D235997 } \\
\text { D235999 } \\
\text { D235998 }\end{array}$ & $\begin{array}{c}9868-9875 \\
10,889-10,904 \\
11,005-11,014\end{array}$ & $\begin{array}{l}4.53 \\
2.93 \\
17.1\end{array}$ & $\begin{aligned} & 9.19 \\
< & 2.6 \\
< & 6.3\end{aligned}$ & $\begin{array}{r}2.03 \\
<0.89 \\
<0.37\end{array}$ & $\begin{array}{l}0.74 \\
1.39 \\
2.13\end{array}$ & $\begin{array}{l}\text { Sisquoc Formation (dolomite) } \\
\text { Monterey Formation } \\
\text { Monterey Formation }\end{array}$ \\
\hline $\begin{array}{l}\text { Newlove } 51 \\
\text { (sec. } 25, \mathrm{~T} .9 \mathrm{~N} . \text {, } \\
\text { R. } 34 \text { W.) }\end{array}$ & $\begin{array}{l}\text { D235980 } \\
D 235981 \\
D 235982 \\
D 235983 \\
D 235984 \\
D 235985 \\
D 235986 \\
D 235987 \\
D 235988 \\
D 235989 \\
D 235990 \\
D 235991 \\
D 235992 \\
D 235993 \\
D 235994 \\
D 235995 \\
D 235996\end{array}$ & $\begin{array}{l}1395-1416 \\
1783-1798 \\
1937-1955 \\
1980-2002 \\
1980-2002 \\
2018-2033 \\
2119-2137 \\
2154-2165 \\
2240-2267 \\
2262-2276 \\
2435-2443 \\
2625-2641 \\
2660-2677 \\
2677-2696 \\
3451-3460 \\
3475-3491 \\
3491-3509\end{array}$ & $\begin{array}{c}2.79 \\
4.32 \\
11.6 \\
14.5 \\
12.2 \\
8.08 \\
13.1 \\
13.1 \\
20.6 \\
15.8 \\
32.2 \\
16.5 \\
14.0 \\
3.02 \\
3.78 \\
4.13 \\
4.07\end{array}$ & $\begin{array}{c}5.6 \\
10.3 \\
<6.6 \\
<6.6 \\
<6.6 \\
<4.8 \\
13.8 \\
14.5 \\
<7.9 \\
<6.8 \\
<10 \\
<6.7 \\
<6.2 \\
5.8 \\
11.7 \\
6.9 \\
8.79\end{array}$ & $\begin{array}{r}2.01 \\
2.38 \\
<0.57 \\
<0.46 \\
<0.54 \\
<0.59 \\
1.05 \\
1.11 \\
<0.38 \\
<0.43 \\
<0.31 \\
<0.41 \\
<0.44 \\
1.81 \\
3.10 \\
1.67 \\
2.16\end{array}$ & $\begin{array}{c}0.86 \\
0.91 \\
3.45 \\
4.15 \\
3.85 \\
4.53 \\
9.26 \\
7.47 \\
7.24 \\
6.40 \\
12.7 \\
9.48 \\
7.85 \\
4.05 \\
2.22 \\
2.11 \\
1.87\end{array}$ & $\begin{array}{l}\text { Sisquoc Formation (clay rich) } \\
\text { Sisquoc Formation (clay rich) } \\
\text { Monterey Formation (cherty zone) } \\
\text { Monterey Formation (cherty zone) } \\
\text { Monterey Formation (cherty zone) } \\
\text { Monterey Formation (cherty zone) } \\
\text { Monterey Formation (cherty zone) } \\
\text { Monterey Formation (bentonitic) } \\
\text { Monterey Formation (bentonitic) } \\
\text { Monterey Formation (bentonitic) } \\
\text { Monterey Formation (bentonitic) } \\
\text { Monterey Formation (bentonitic) } \\
\text { Monterey Formation (bentonitic) } \\
\text { Monterey Formation (bentonitic) } \\
\text { Point Sal Formation } \\
\text { Point Sal Formation } \\
\text { Point Sal Formation }\end{array}$ \\
\hline
\end{tabular}


Eight samples of Monterey Formation from cores from near the Temblor Range, and 22 samples of Monterey and related formations from cores from near Santa Maria were analyzed for uranium and thorium (tables 5, 6). The results show that the Monterey, in these two areas at least, has as much or more uranium in the subsurface than it generally does at the surface. Also, the thorium-uranium ratios of the core samples generally are lower than ratios common in outcrop samples.

The 22 core samples from near Santa Maria also were analyzed for organic carbon (table 6). The organic carbon content of 16 samples from the Monterey Formation ranged from 1.39 to 14.4 percent, averaged 6.36 percent, and had a median value near 6 percent.

The uranium content of seven samples of diatomite from cores of rocks correlative with the Monterey Formation taken on the California Continental Borderland ranged from about 7 to $41 \mathrm{ppm}$ (table 7), values considerably higher than for most outcrop samples of the Monterey. On the other hand, the uranium content of lower Miocene diatom ooze from the Bering Sea (table
8), and of siliceous sediments from the equatorial Pacific (table 9) generally are less than $3 \mathrm{ppm}$, considerably less than for most rocks of the Monterey. However, 19 samples of siliceous rocks from South America and Mexico were analyzed and found to have about the same uranium content as do similar rocks in the Monterey (table 10).

\section{Interpretation of Analytical Data}

If the samples from uranium prospects in the Temblor Range are omitted, samples of Monterey Formation analyzed show no systematic regional variation in uranium content. This seems to show that conditions that caused the accumulation of uranium prevailed wherever diatomaceous sediments of the Monterey formed; variations in uranium content depended on factors other than location. This suggestion is supported by the similarity of the uranium content of diatomite and porcelanite from Mexico and Peru to that of similar rocks

Table 7. Uranium and thorium content of diatomite in cores taken on California Continen-

tal Borderland

[Samples provided by J. R. Hein. ---, leaders indicate no data]

\begin{tabular}{|c|c|c|c|c|c|c|c|}
\hline Field No. & Lab. No. & Lat. N & Long. W & $\underset{(p p m)}{U}$ & $\begin{array}{c}\text { Th } \\
(\mathrm{ppm})\end{array}$ & $\begin{array}{c}\mathrm{eU} \\
(\mathrm{ppm})\end{array}$ & Th/U \\
\hline L2-78-SC-29 & D226938 & $33^{\circ} 51^{\prime} 42^{\prime \prime}$ & $119^{\circ} 28^{\prime} 12^{\prime \prime}$ & 19.8 & $<19$ & -- & $<0.96$ \\
\hline L2-78-SC-123 & D226939 & $32^{\circ} 21^{\prime} 48^{\prime \prime}$ & $118^{\circ} 24^{\prime} 42^{\prime \prime}$ & 41.6 & $<28$ & 30 & $<0.67$ \\
\hline L2-78-SC-134 & D226940 & $32^{\circ} 19^{\prime} 24^{\prime \prime}$ & $118^{\circ} 52^{\prime} 24^{\prime \prime}$ & 17.5 & $<16$ & $<10$ & $<0.91$ \\
\hline L2-78-SC-158 & D226941 & $32^{\circ} 30^{\prime} 12^{\prime \prime}$ & $119^{\circ} 19^{\prime} 06^{\prime \prime}$ & 9.36 & $<10$ & 20 & $<1.07$ \\
\hline L2-78-SC-179 & D226942 & $32^{\circ} 32^{\prime} 54^{\prime \prime}$ & $119^{\circ} 37^{\prime} 12^{\prime \prime}$ & 7.17 & $<11$ & 20 & $<1.53$ \\
\hline L2-78-SC-184 & D226943 & $322^{\prime} 24^{\prime \prime}$ & $119^{\circ} 41^{\prime} 12^{\prime \prime}$ & 20.0 & $<16$ & 10 & $<0.80$ \\
\hline L2-78-SC-322 & D226944 & $33^{\circ} 50^{\prime} 12^{\prime \prime}$ & $119^{\circ} 53^{\prime}$ & 24.2 & $<15$ & 60 & $<0.62$ \\
\hline
\end{tabular}

Table 8. Uranium and thorium content of lower Miocene diatom ooze from Bering Sea [Samples provided by J. R. Hein. ---, leaders indicate no data]

\begin{tabular}{|c|c|c|c|c|c|c|c|c|}
\hline Field No. & Lab. No. & Lat. N & Long. W & $\underset{(p p m)}{U}$ & $\begin{array}{c}\text { Th } \\
\text { (ppm) }\end{array}$ & $\begin{array}{c}\mathrm{eU} \\
(\mathrm{ppm})\end{array}$ & $\mathrm{Th} / \mathrm{U}$ & Remarks \\
\hline $19-184-7-1-110-112$ & D226945 & $53^{\circ} 42^{\prime} 38^{\prime \prime}$ & $170^{\circ} 55^{\prime} 23^{\prime \prime}$ & 2.31 & $<5.5$ & 10 & $<2.38$ & Silt and clay bearing. \\
\hline $19-184-10-2-118-120$ & D226946 & $53^{\circ} 42^{\prime} 38^{\prime \prime}$ & $170^{\circ} 55^{\prime} 23^{\prime \prime}$ & 2.48 & 6.6 & $<10$ & 2.66 & -- \\
\hline $19-184-17-2-86-88$ & D226947 & $53^{\circ} 42^{\prime} 38^{\prime \prime}$ & $170^{\circ} 55^{\prime} 23^{\prime \prime}$ & 2.01 & 7.8 & 10 & 3.88 & Silt bearing, clay rich. \\
\hline $19-185-6-3-95-97$ & D226948 & $53^{\circ} 25^{\prime} 44^{\prime \prime}$ & $169^{\circ} 14^{\prime} 35^{\prime \prime}$ & 1.43 & $<5.5$ & 10 & $<3.85$ & Silt bearing, clay rich. \\
\hline $19-188-2-1-120-124$ & D226949 & $54^{\circ} 25^{\prime} 44^{\prime \prime}$ & $169^{\circ} 14^{\prime} 35^{\prime \prime}$ & 2.56 & $<9.1$ & 10 & $<3.55$ & Silt, clay, and spicule bearing. \\
\hline $19-188-8-6-145-147$ & D226950 & $54^{\circ} 25^{\prime} 44^{\prime \prime}$ & $169^{\circ} 14^{\prime} 35^{\prime \prime}$ & 1.5 & $<7.5$ & 20 & $<5.00$ & -- \\
\hline $19-188-15-2-134-136$ & D226951 & $54^{\circ} 25^{\prime} 44^{\prime \prime}$ & $169^{\circ} 14^{\prime} 35^{\prime \prime}$ & $<0.59$ & $<5.0$ & $<10$ & $<8.47$ & Silt and clay bearing. \\
\hline $19-190-1-1-48-50$ & D226952 & $55^{\circ} 33^{\prime} 33^{\prime \prime}$ & $17138^{\prime} 25^{\prime \prime}$ & 1.47 & $<5.6$ & 20 & $<3.81$ & -- \\
\hline $19-190-13-1-105-108$ & D226953 & $55^{\circ} 33^{\prime} 33^{\prime \prime}$ & $1711^{\circ} 8^{\prime} 25^{\prime \prime}$ & 2.17 & $<6.4$ & $<10$ & $<2.95$ & Silt and clay bearing. \\
\hline $19-192-4-3-130-132$ & D226954 & $53^{\circ} 00^{\prime} 34^{\prime \prime}$ & $164^{\circ} 42^{\prime} 49^{\prime \prime}$ & 1.65 & 7.4 & 40 & 4.48 & Silt, clay, and carbonate bearing. \\
\hline $19-192-14-3-40-42$ & D226955 & $53^{\circ} 00^{\prime} 34^{\prime \prime}$ & $164^{\circ} 42^{\prime} 49^{\prime \prime}$ & 2.51 & $<6.4$ & 10 & $<2.55$ & Silt and clay rich. \\
\hline $19-192-20-1-100-104$ & D226556 & $53^{\circ} 00^{\prime} 34^{\prime \prime}$ & $164^{\circ} 42^{\prime} 49^{\prime \prime}$ & 3.14 & $<5.9$ & 20 & $<1.88$ & Silt bearing, clay rich. \\
\hline
\end{tabular}


Table 9. Uranium and thorium content of siliceous sediments from the equatorial Pacific [Samples provided by J. R. Hein. ---, leaders indicate no data]

\begin{tabular}{|c|c|c|c|c|c|c|c|c|}
\hline Field No. & Lab. No. & Lat. $\mathrm{N}$ & Long. W & $\underset{(\mathrm{ppm})}{U}$ & $\begin{array}{l}\text { Th } \\
(\mathrm{ppm})\end{array}$ & $\begin{array}{c}\mathrm{eU} \\
(\mathrm{ppm})\end{array}$ & Th/U & Remarks \\
\hline $46-1-9-12 \mathrm{CM}-\mathrm{A}$ & D226977 & $90^{\prime} 42^{\prime \prime}$ & $150^{\circ} 50^{\prime} 42^{\prime \prime}$ & 2.32 & 11. & --- & 4.74 & $\begin{array}{l}\text { Glass-bearing, clayey siliceous } \\
\text { ooze, } 5160 \mathrm{~m} \text { depth. }\end{array}$ \\
\hline $46-1-9-12 C M-B$ & D226978 & $90^{\prime} 42^{\prime \prime}$ & $150^{\circ} 50^{\prime} 42^{\prime \prime}$ & 1.8 & 22. & 60 & 12.2 & Same, $>44 \mu$ size fraction. \\
\hline $52-42-4-7 \mathrm{CM}-\mathrm{A}$ & D226979 & $11^{\circ} 15^{\prime} 30^{\prime \prime}$ & $139^{\circ} 03^{\prime} 18^{\prime \prime}$ & 2.13 & 12. & -- & 5.63 & Siliceous mud, $4871 \mathrm{~m}$ depth. \\
\hline $52-42-4-7 \mathrm{CM}-\mathrm{B}$ & D226980 & $11^{\circ} 15^{\prime} 30^{\prime \prime}$ & $139^{\circ} 03^{\prime} 18^{\prime \prime}$ & $<2.5$ & $<24$ & -- & -- & Same $>44 \mu$ size fraction. \\
\hline $48-19-22-26 A$ & D226981 & $8^{\circ} 16^{\prime} 30^{\prime \prime}$ & $151^{\circ} 07^{\prime} 30^{\prime \prime}$ & 1.67 & 11.3 & 60 & 6.7 & $\begin{array}{l}\text { Glass-bearing, siliceous, fossil- } \\
\text { rich mud, } 5045 \mathrm{~m} \text { depth. }\end{array}$ \\
\hline $48-19-22-26 B$ & D226982 & $8^{\circ} 16^{\prime} 30^{\prime \prime}$ & $151^{\circ} 07^{\prime} 30^{\prime \prime}$ & $<4.0$ & $<40$ & --- & -- & Same $>44 \mu$ size fraction. \\
\hline
\end{tabular}

of the Monterey. In other words, rocks similar to those in the Monterey have a similar range of uranium content, regardless of where they formed.

The uranium content of diatomite, porcelanite, and calcareous mudstone samples from the Monterey Formation-except samples from uranium prospectswere all in the same general range of values, but the uranium content of the few chert samples analyzed was lower. This difference suggests that uranium was lost during the later stages of diagenesis of Monterey rocks. To determine whether this apparent loss of uranium is gradual or abrupt, the uranium content of 29 samples of porcelanite was compared with the degree of diagenesis of the rock (table 11). The degree of diagenesis can be approximated from the ordering of cristobalite as expressed by the height-to-width ratio of the (101) cristobalite peak obtained by X-ray diffractometry of whole-rock samples (fig. 9). For samples that contain less than $15 \mathrm{ppm}$ uranium - that is, samples that probably have not been enriched appreciably beyond their original uranium content-the uranium content generally decreases with an increase in the degree of diagenesis (fig. 10). This relationship suggests that uranium may be lost during diagenesis of porcelanite, and presumably this uranium would be available in mobile form for enrichment of other rocks.

Water-depth changes during Miocene time are not reflected in any trend of the uranium content of rocks

Table 10. Uranium and thorium content of siliceous rocks from South America and Mexico

[Sample collected by J. R. Hein. Abbreviations for rocks: ch, chert; di, diatomite; pc, cherty porcelanite; po, porcelanite]

\begin{tabular}{|c|c|c|c|c|c|c|c|c|c|}
\hline Field No. & Lab. No. & Lat. & Long. W & Rock & $\underset{(p p m)}{U}$ & $\begin{array}{c}\text { Th } \\
\text { (ppm) }\end{array}$ & $\begin{array}{c}\mathrm{eU} \\
(\mathrm{ppm})\end{array}$ & $\mathrm{Th} / \mathrm{U}$ & Remarks \\
\hline $979-13-1 \mathrm{~A}$ & D226957 & $25^{\prime} \mathrm{S}$ & $80^{\circ} 45^{\prime}$ & $\operatorname{ch}$ & $<0.23$ & $<2.2$ & 10 & -- & Miocene(?), Equador. \\
\hline $979-13-2 C$ & D226958 & $25^{\prime} \mathrm{S}$ & $80^{\circ} 45^{\prime}$ & ch & 0.80 & $<2.6$ & 10 & $<3.25$ & Miocene(?), Equador. \\
\hline $979-15-1$ & D226959 & $23^{\circ} \mathrm{S}$ & $80^{\circ} 45^{\prime}$ & ch & 1.41 & $<3.1$ & 10 & $<2.20$ & Miocene(?), Equador. \\
\hline $979-18-1 \mathrm{~A}$ & D226960 & $14^{\circ} 35^{\prime} \mathrm{S}$ & $75^{\circ} 45^{\prime}$ & di & 6.04 & 17.7 & 20 & 2.93 & Miocene(?), Peru. \\
\hline $979-18-1 B$ & D226961 & $14^{\circ} 35^{\prime} \mathrm{S}$ & $75^{\circ} 45^{\prime}$ & di & 5.42 & 14.4 & 60 & 2.65 & Miocene(?), Peru. \\
\hline $979-18-2 B$ & D226962 & $14^{\circ} 30^{\prime} \mathrm{S}$ & $75^{\circ} 45^{\prime}$ & di & 4.69 & 16.9 & 30 & 3.60 & Miocene(?), Peru. \\
\hline $979-18-2 C$ & D226963 & $14^{\circ} 30^{\prime} \mathrm{S}$ & $75^{\circ} 45^{\prime}$ & di & 4.13 & 11. & 10 & 2.66 & Miocene(?), Peru. \\
\hline $979-18-3$ & D226964 & $14^{\circ} 35^{\prime} \mathrm{S}$ & $75^{\circ}$ & di & 5.78 & $<8.6$ & 20 & $<1.49$ & Miocene(?), Peru. \\
\hline $979-18-4 B$ & D226965 & $13^{\circ} 45^{\prime} \mathrm{S}$ & $76^{\circ} 25^{\prime}$ & di & 49.8 & $<30$ & 40 & $<0.60$ & Miocene(?), Peru. \\
\hline $979-18-4 C$ & D226966 & $13^{\circ} 45^{\prime} \mathrm{S}$ & $76^{\circ} 25^{\prime}$ & di & 19.2 & $<14$ & $<10$ & $<0.73$ & Miocene(?), Peru. \\
\hline 979-18-4D & D226967 & $13^{\circ} 45^{\prime} \mathrm{S}$ & $76^{\circ} 25^{\prime}$ & di & 14.0 & $<15$ & 40 & $<1.07$ & Miocene(?), Peru. \\
\hline $979-18-4 \mathrm{E}$ & D226968 & $13^{\circ} 45^{\prime} \mathrm{S}$ & $76^{\circ} 25^{\prime}$ & di & 2.67 & 9.9 & 30 & 3.71 & Miocene(?), Peru. \\
\hline H6-1C & D226969 & $31^{\circ} 05^{\prime} \mathrm{N}$ & $115^{\circ} 05^{\prime}$ & di & 5.64 & $<9.3$ & 10 & $<1.65$ & Miocene-Pliocene, Baja Calif. \\
\hline H6-1D & D226970 & $31^{\circ} 05^{\prime} \mathrm{N}$ & $115^{\circ} 05^{\prime}$ & di & 5.31 & 8.4 & 20 & 1.58 & Miocene-Pliocene, Baja Calif. \\
\hline H6-2A & D226971 & $31^{\circ} 05^{\prime} \mathrm{N}$ & $115^{\circ} 05^{\prime}$ & di & 6.21 & $<10$ & 20 & $<1.61$ & Miocene-Pliocene, Baja Calif. \\
\hline H6-2B & D226972 & $31^{\circ} 05^{\prime} \mathrm{N}$ & $115^{\circ} 05^{\prime}$ & di & 6.50 & $<9.1$ & 30 & $<1.40$ & Miocene-Pliocene, Baja Calif. \\
\hline H6-2E & D226973 & $31^{\circ} 05^{\prime} \mathrm{N}$ & $115^{\circ} 05^{\prime}$ & di & 7.63 & $<12$ & 40 & $<1.57$ & Miocene-Pliocene, Baja Calif. \\
\hline 1179-1-1A & D226974 & $2420^{\prime} \mathrm{N}$ & $111^{\circ} 02^{\prime}$ & pc & 2.23 & $<4.2$ & 20 & $<1.88$ & Miocene, Baja Calif. \\
\hline 1179-1-1B & D226975 & $24^{\circ} 20^{\prime} \mathrm{N}$ & $111^{\circ} 02^{\prime}$ & po & 3.19 & $<4.7$ & 10 & $<1.47$ & Miocene, Baja Calif. \\
\hline
\end{tabular}



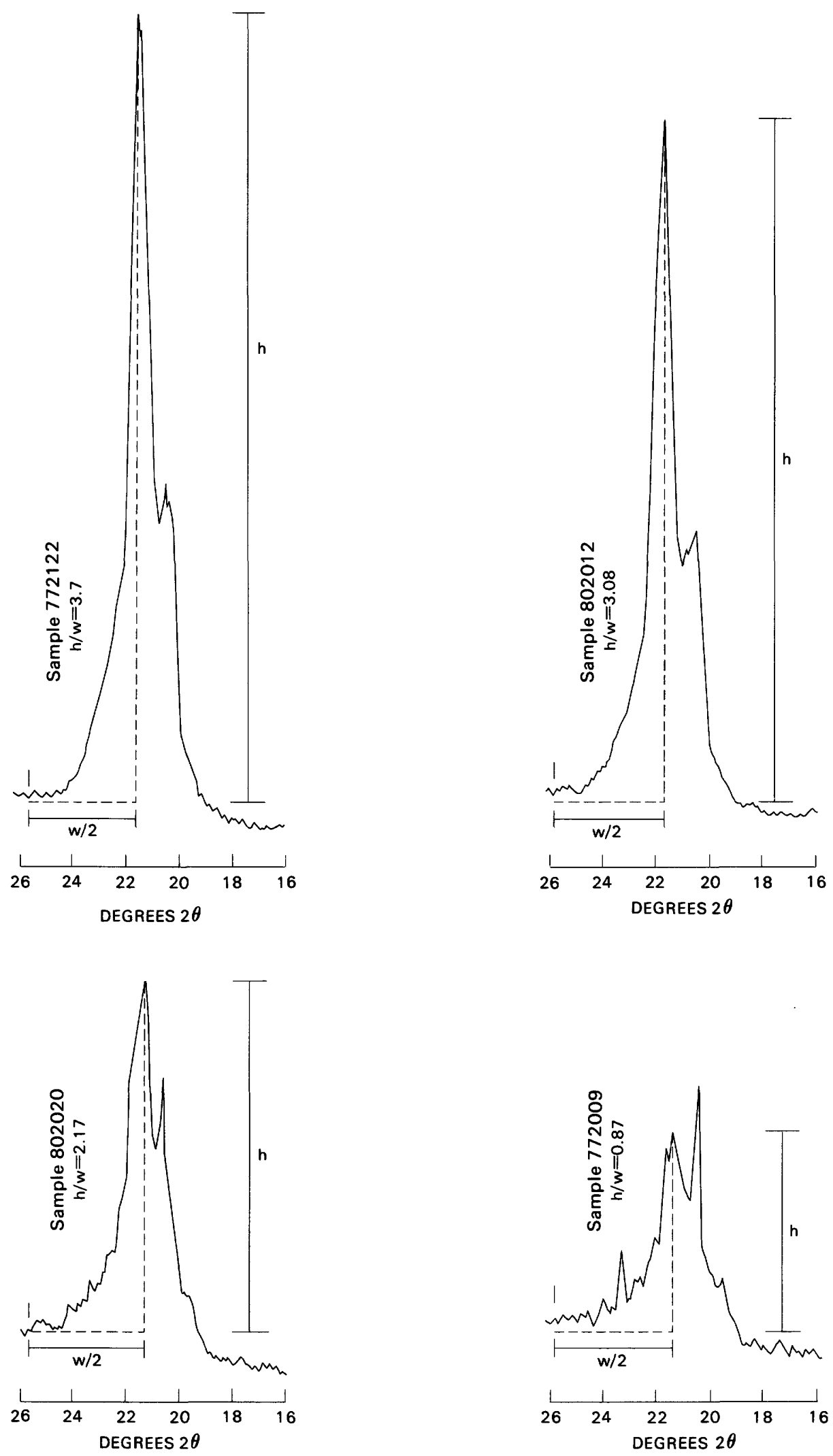

Figure 9. Typical X-ray diffractograms of porcelanite showing various height-to-width ratios $(\mathrm{h} / \mathrm{w})$ of $(101)$ cristobalite peak. Such ratios help to determine the degree of diagenesis of the rock. 
Table 11. Height-to-width ratio of the (101) cristobalite peak obtained by X-ray diffractometry of samples of porcelaneous rock

\begin{tabular}{|c|c|c|}
\hline Sample No. & height/width & $U(p p m)$ \\
\hline 764003 & 4.10 & 4.37 \\
\hline 771080 & 2.24 & 9.34 \\
\hline 772009 & 0.87 & 14.3 \\
\hline 772122 & 3.7 & 4.40 \\
\hline 772139 & 2.17 & 7.99 \\
\hline 784005 & 1.37 & 11.5 \\
\hline 784008 & 2.79 & 10.7 \\
\hline 784014 & 3.26 & 8.71 \\
\hline 784017 & 3.14 & 4.13 \\
\hline 784018 & 3.14 & 9.65 \\
\hline 802004 & 2.78 & 8.95 \\
\hline 802007 & 2.62 & 5.34 \\
\hline 802009 & 2.20 & 8.06 \\
\hline 802010 & 1.37 & 8.56 \\
\hline 802011 & 3.91 & 6.30 \\
\hline 802012 & 3.08 & 6.65 \\
\hline 802013 & 3.22 & 7.07 \\
\hline 802014 & 2.84 & 2.48 \\
\hline 802015 & 1.89 & 10.2 \\
\hline 802016 & 3.55 & 5.04 \\
\hline 802020 & 2.17 & 9.93 \\
\hline 802021 & 2.00 & 12.2 \\
\hline 802022 & 3.22 & 11.3 \\
\hline 802025 & 2.17 & 6.02 \\
\hline 802029 & 3.87 & 2.40 \\
\hline 802030 & 3.48 & 6.80 \\
\hline 802036 & 2.57 & 12.7 \\
\hline 802040 & 4.28 & 4.72 \\
\hline 802049 & 3.14 & 5.39 \\
\hline
\end{tabular}

of the Monterey Formation, which suggests that water depth was less important than other factors in the accumulation of uranium in the sediments that formed the Monterey. However, details concerning the conditions of deposition of most of the samples are too few to allow any firm conclusions regarding the relation of depositional environment to uranium content. On the other hand, it does seem likely that persistence of the anoxic conditions commonly associated with the deposition of diatomaceous sediments should be an important consideration.

Comparison of the uranium and thorium contents of samples from the Monterey Formation provides some insight into the source of the uranium found in the formation. A thorium-uranium ratio of 3 or 4 is characteristic of most rocks in the Earth's crust-and of sediments composed of debris from these rocks. On the other hand, sea water and water in streams that feed the sea have a very low ratio of dissolved thorium to uranium (Adams and Weaver, 1958, p. 429). This low ratio reflects the much greater solubility of uranium compared to thorium, and consequently the greater amount of uranium that goes into solution during weathering of terrestrial rocks. Adams and Weaver $(1958$, p. 415$)$ concluded that a low $(<2)$ thorium-uranium ratio in sediments or sedimentary rocks indicates that most of the uranium in the sediment or rock was extracted from water rather than contributed by detrital matter derived from rocks. Therefore, the generally low-2 or less-thorium-uranium ratio in rocks of the Monterey Formation (tables 1, 3, 5, and 6) suggests that most of the uranium was derived from sea water. Such a conclusion is reasonable when it is realized 


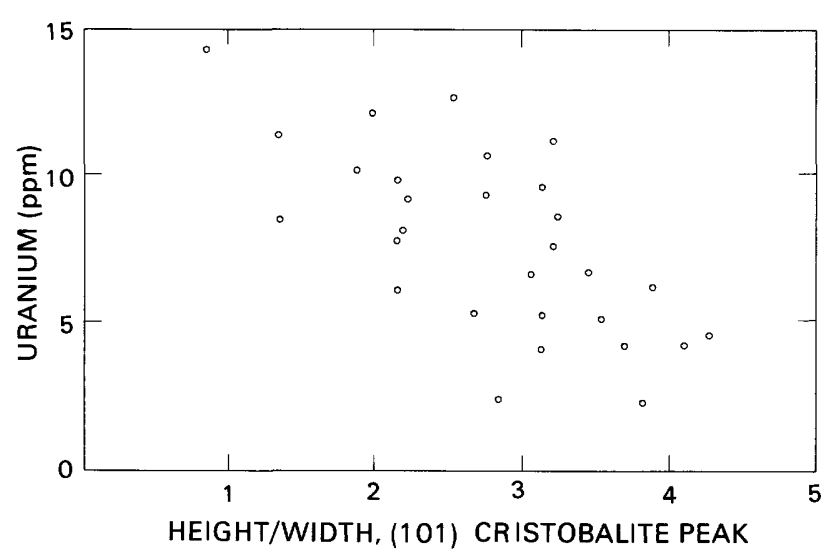

Figure 10. Graph showing relation of uranium content to stage of diagenesis of porcelanite samples. (The degree of diagenesis is represented by the ordering of diagenetic cristobalite as expressed by the height-to-width ratio of the (101) cristobalite peak obtained by X-ray diffractometry; table 11.)

that nearly all the substance of pure diatomaceous rocks was derived from sea water. It also is compatible with the observation that some of the highest thorium-uranium values found in the Monterey occur in rocks such as mudstone that have a substantial proportion of terrigenous debris. Moreover, the commonly lower thorium/uranium values found in core samples, as compared to surface samples, can be interpreted as evidence that some uranium has been leached from the surface rocks during weathering.

Samples of diatom ooze from the Bering Sea (table 8) and of siliceous sediments from the equatorial Pacific (table 9) have higher thorium-uranium ratios than are common in rocks of the Monterey Formation. However, these oceanic sediments formed under conditions quite different from conditions under which Monterey sediments accumulated. In fact, these oceanic sediments have a low uranium content, and their high thorium-uranium ratios indicate that what little uranium they have came from terrestrial particulate material rather than from sea water. By contrast, the thorium-uranium ratios of most samples of Montereylike rocks from South America and Mexico (table 10) are similar to the ratios for rocks from the Monterey Formation, which may suggest a similar, sea-water origin for the uranium in all Montereylike rocks.

The samples of pellet phosphorite from the Monterey Formation that were analyzed for uranium (samples 784009 and 802006) have, as expected, a higher uranium content than do most Monterey rocks. This comparatively high uranium content is easily attributed to the well-known affinity of uranium for phosphate minerals. However, the lack of any correlation between uranium and phosphorus in other kinds of Monterey rock that were analyzed for both elements (table 4) shows that little or none of the uranium in these other rocks is involved with phosphate minerals.

The general increase in uranium content with increase in organic-carbon content of core samples of Monterey Formation (fig. 11) suggests that at least an appreciable part of the uranium in the samples is associated in some way with organic material. This is consistent with the work of Burton and Sullivan (1951, p. 884), who found an excellent correlation between carbon and uranium contents of Miocene shale from the Lawndale oil field near Los Angeles. Conversely, Ross (1952, p. 791) found that the radioactivity of Miocene shale from the Playa del Rey oil field, also near Los Angeles, is localized in inorganic nodules and layers, particularly in phosphatic material, rather than in organic layers and veinlets.

Although rocks of the Monterey Formation and equivalent beds generally are considered to be the principal source of oil in the reservoir rocks of California oil fields, California crude oil is not especially high in uranium. The uranium content of 11 samples of crude oil from Tertiary rocks of California given by Bell (1960, table 2) ranged from 0.1 to 37.7 parts per billion (ppb), averaged $5.04 \mathrm{ppb}$, and had a median value of $1.6 \mathrm{ppb}$. This compares to the range for crude oil generally from less than $1 \mathrm{ppb}$ to a few tens ppb (Bell, 1960, p. 51). However, asphalt-bearing rocks of California contain more uranium than do most such rocks, and the asphalt likely originated in the Monterey Formation (Hail and others, 1956, p. 524; Bell, 1960; p. 54). Bell (1960, table 3) listed the uranium contents of 51 samples of rock asphalt from California Tertiary rocks; the uranium

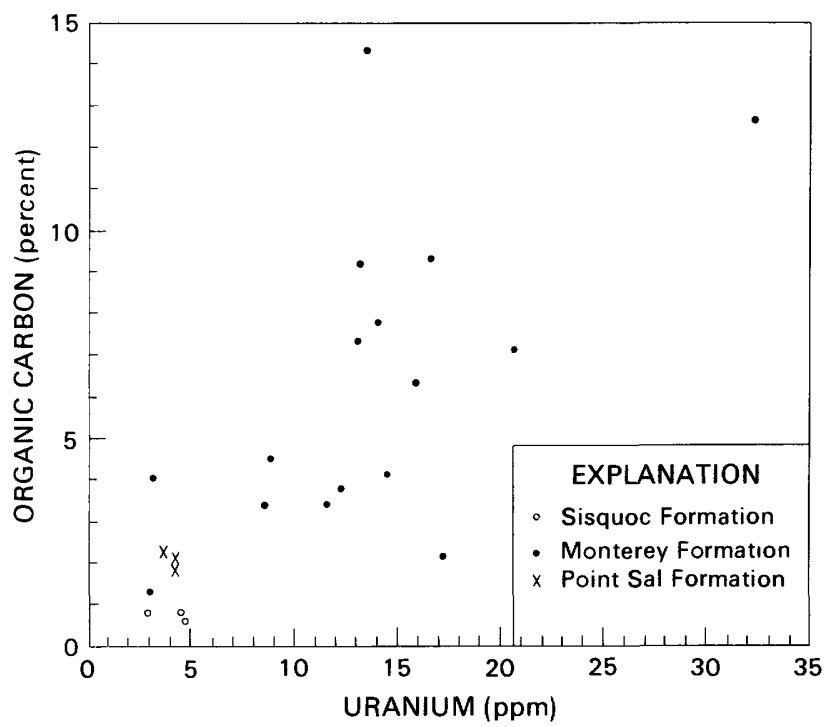

Figure 11. Graph showing relation of uranium content to organic-carbon content of core samples from Monterey Formation and related rocks near Santa Maria. 
content ranged from 0.17 to $70.3 \mathrm{ppm}$, , averaged 5.22 $\mathrm{ppm}$, and had a median value of $1.6 \mathrm{ppm}$. These figures show that uranium is not concentrated more in rock asphalt than it is in the Monterey.

Although a connection between the uranium and organic-carbon contents of rocks from the Monterey Formation appears probable, it is difficult to consider petroleum to be responsible for the uranium in the formation, for the uranium content of both crude oil and asphalt are less than the uranium content of most Monterey rocks. To account for the uranium in the Monterey it seems best to review aspects of uranium in the marine environment. A considerable amount of information on uranium in sea water and in marine sediments is available.

\section{URANIUM IN THE MARINE ENVIRONMENT}

\section{Sea Water}

\section{Uranium Content}

Starik and Kolyadin (1957, p. 247) indicated that uranium occurs in sea water (1) in the colloidal state, (2) adsorbed on colloidal particles, and (3) as dispersed ions, including complexes with organic components of the water. Calculations concerning uranium supplied to and removed from the sea suggest that a steady state with respect to uranium is possible in the world ocean (Bloch, 1980 , p. 376). The calculations involve both particulate and dissolved uranium, but it seems likely that the dissolved uranium content of sea water does not vary greatly.

Goldberg $(1963$, p. 5) gave the uranium content of sea water as $3 \mathrm{ppb}$. Ku, Knauss, and Mathieu (1977) reviewed previous determinations and presented the results of their own analyses of $\mathbf{6 3}$ water samples from the Atlantic, Pacific, Arctic, and Antarctic Oceans. The uranium content of their samples ranged from 2.9 to $3.6 \mathrm{ppb}$; they $(1977$, p. 1010) attributed the range to variation in the salinity of the water. They $(1977$, p. 1012) also determined the average uranium concentration of water in the open ocean (corrected to 35 parts per thousand salinity) to be $3.3 \pm 0.2 \mathrm{ppb}$. Hodge, Koide, and Goldberg $(1979$, p. 207) showed that some of the uranium in sea water is in particulate form, but $\mathrm{Ku}$, Knauss, and Mathieu (1977, p. 1011) considered that nearly all is in the dissolved state. Sea water, then, seems to be an adequate and constant source of uranium, in the same way that sea water is the source of silica for diatomaceous sediments.

\section{Extraction of Uranium}

Evidence from the isotopic composition of uranium in modern marine sediments has been used to demonstrate that uranium presently is being extracted from sea water, but there seems to be no really clear evidence concerning the mechanism of extraction (Burton, 1965, p. 438). Uranium isotopes in sea water are in disequilibrium because ${ }^{234} \mathrm{U}$ goes into solution more readily than does ${ }^{238} \mathrm{U}$. The ${ }^{234} \mathrm{U} /{ }^{238} \mathrm{U}$ activity ratio in river water generally is greater than 1 , and in residual solids it generally is about 1 , or less. Thus, uranium from a terrigenous source in modern marine sediments may be identified by its ${ }^{234} \mathrm{U} /{ }^{238} \mathrm{U}$ activity ratio of less than 1 (Hodge and others, 1979 , p. 207). For example, deep-sea clay has uranium concentrations and ${ }^{234} \mathrm{U} /{ }^{238} \mathrm{U}$ activity ratios similar to those of stream sediments, which shows that the clay is predominantly detrital. On the other hand, uranium concentrations in anoxic mud are much higher than in terrigenous debris, and the ${ }^{234} \mathrm{U} /{ }^{238} \mathrm{U}$ activity ratios for anoxic mud approach the sea-water value of 1.15 , which indicates a sea-water origin for most of the uranium in the mud (Veeh, 1967, p. 147). Veeh, Calvert, and Price $(1974$, p. 193) used this evidence to determine that uranium is passing from sea water into diatomaceous sediments off California, Mexico, Central and South America, and southwest Africa. Application of the ${ }^{234} \mathrm{U} /{ }^{238} \mathrm{U}$ activity ratio to finding the source of uranium is, of course, restricted to comparatively recent sediments.

\section{Role of Organic Carbon}

Although the means by which uranium is extracted from sea water and concentrated in marine sediments is uncertain, most investigators find a positive correlation between the uranium and organic-carbon contents of marine sediments (Baturin, 1968a, p. 345, 1973, p. 1034; Kuznetsov and others, 1968, p. 307; Mo and others, 1971). Conversely, Veeh, Calvert, and Price (1974, p. 193) failed to find such a correlation for diatomaceous sediments off southwest Africa, either because no correlation exists or because it is masked by other factors that control distribution of uranium. Weber and Sackett $(1981$, p. 1328) also failed to find any correlation between uranium and organic carbon in sediments of the anoxic Orca Basin in the northwestern Gulf of Mexico.

Generally, the lowest uranium-organic carbon ratios occur in sandy and silty sediments deposited at relatively high rate, and the highest ratios occur in finegrained sediments enriched in both organic matter and uranium (Baturin, 1974, p. 190). Most deep-water oceanic sediments lack significant uranium enrichment because 
they contain little organic matter and are flushed by oxygenated water (Baturin and others, 1972, p. 281). Langmuir (1977, p. 27) suggested that clays, which are abundant in deep-water oceanic sediments, are relatively unimportant as concentrators of uranium. Balistrieri, Brewer, and Murray (1981, p. 119) suggested that the adsorption properties of particles sinking through the marine water column are controlled by organic coatings on the particles rather than by the properties of the particles themselves.

The general correlation of uranium and organic carbon in marine sediments could mean either that organic material incorporates uranium directly or that it produces a reducing environment favorable for chemical extraction of uranium from sea water. Baturin and Lisitsyn (1973, p. 878) reported experimental evidence that showed that uranium may be captured by organic matter under both anaerobic and aerobic conditions. Organic material that incorporates uranium directly might be in suspension, at the sediment surface, or within the sediments, but the water-sediment interface is considered the most likely place (Baturin, 1968a, p. 345, 347).

Baturin (1973, p. 1034) reported that uranium is not concentrated by living tissues, and thus extraction of uranium from sea water by organic material probably takes place not from the water column as a whole, but only at the bottom. But Degans, Khoo, and Michaelis (1977, p. 568) reported that in Black Sea sediments, uranium seems to be bound to planktonic rather than to land-derived organic debris. They tentatively concluded that certain organic compounds act as a substrate in the formation of hexavalent uranium complexes and concentrate uranium in the living cell as much as 10,000 times the uranium concentration in sea water. Thus, although reducing conditions may be needed for preservation of uranium-enriched debris, they may not be needed for fixation of uranium. Holland (1979, p. 1679) concluded that concentration of metals in organic-rich sediments apparently results more from chemical precipitation and from reactions with dead organic remains than from their incorporation in living organisms. Apparently, marine organisms can absorb uranium from sea water, and the concentration factor may be considerable (Zverev and others, 1976, p. 91), but the importance of organisms in extraction of uranium and the means by which they do it is uncertain.

Kuznetsov, Simonyak, Lisitsyn, and Frenklikh (1968, p. 307) concluded that there is little probability of biogenic extraction of uranium by suspended organic matter because they found little correlation between uranium and organic carbon in material collected from the water column in the Indian Ocean; they did find a direct correlation between uranium and organic carbon in seafloor sediments, and they allowed that dissolved organic matter might interact with dissolved uranium, perhaps by ion exchange or chelation, and thereby transfer uranium to the bottom. Although the enhancement of uranium in sediments in anoxic regimes is well established, good evidence is lacking that reduction of uranium in the overlying water column contributes to the process (Burton, 1975, p. 102). More likely, uranium is removed from the sea water by reduction and sorption on organic matter on the sea floor (Baturin, 1969, p. 831; Baturin and others, 1976, p. 90; Kuznetsov and others, 1968, p. 310). Langmuir $(1978$, p. 565$)$ considered that uranium after adsorption may be reduced to the $\mathrm{U}^{+4}$ of uraninite or coffinite by mobile reductants, such as hydrogen sulfide or methane, or by the sorbent itself. $\mathrm{He}(1977$, p. 27) found good evidence that uranyl sorption is an important preconcentrating step leading to the formation of both uranyl and uranous minerals.

Baturn, Kochenov, and Kovaleva (1966, p. 174) explained a depletion of uranium in bottom water of the Black Sea by loss of uranium to the sediments, and Baturin (1968a, p. 345) concluded that the principal factor in the extraction of uranium by sediments is the geochemical activity of organic material-the presence of hydrogen sulfide being only a marginal factor. Baturin $(1974$, p. 192) later generalized that the process by which uranium is concentrated in organic material in sediments is the same in basins with normal aeration as in basins with a hydrogen-sulfide environment. He then attributed the only slightly higher uranium-organic carbon ratio of anoxic sediments of the Black Sea, as compared to the ratio in sediments of normally aerated marine basins, to the extremely sluggish circulation of bottom water in the Black Sea. In other words, the enrichment of uranium in sediments depends, even under the best of other conditions on a continued supply of water undepleted in uranium. Baturin, Lebedev, and Mayev (1976, p. 89) considered that the accumulation of uranium in organic-rich sediments is independent of the hydrogen sulfide content of the water-the hydrogen sulfide only affects the completeness with which uranium is extracted from the bottom layer of water.

The presence of hydrogen sulfide in diatomaceous and other highly organic sediments is common and indicates an anoxic regime. Samples of diatomaceous ooze taken from water depths greater than $60 \mathrm{~m}$ on the southwest African shelf had a pronounced odor of hydrogen sulfide, lacked an oxidized zone at the ocean floor surface, and had no sign of a benthonic fauna; these conditions indicate that below $60 \mathrm{~m}$ depth hydrogen sulfide there passes from the sediment into and remains in the 
bottom water, unlike at shallower depths where wave action aerates bottom water (Baturin and others, 1972, p. 282). But even in the highly anoxic Black Sea water Eh values are too high to allow precipitation of uranium by reduction of hexavalent to tetravalent uranium; however, interstitial waters in the upper layer of sediment may have Eh values low enough to allow such precipitation (Pokidin and others, 1972, p. 570). Ku, Knaus, and Mathieu (1977, p. 1013) found that the uranium content of pore water generally is close to, or slightly enriched over that of sea water, although Baturin (1971a, p. 224) noted that during early diagenesis of sediments uranium passes into interstitial water, where its concentration may be tens or hundreds of times higher than in sea water. Regardless of conditions respecting pore water, the principal carriers of uranium in the sediment seems to be the components with maximum sorptive capacity-organic matter and phosphates (Kochenov and others, 1977, p. 86). Uranium is mobile during diagenesis of sediments, and apparently part of the uranium is in solution in the form of organometallic complexes (Baturin and Kochenov, 1973).

Bonatti, Fisher, Joensuu, and Rydell (1971) described a core of deep-sea hemipelagic sediment from off South America that had an upper oxidized zone above reduced sediments, and the upper oxidized zone is enriched in uranium, chromium, vanadium, and sulphur. They explained distribution of uranium in the core by the postdepositional mobility of uranium, mainly by diffusion in the interstitial solutions. They found no abrupt change in uranium concentration across the Eh interface in the core, but they noted a steady increase of uranium with depth in the reduced part of the core (1971, p. 196). They interpreted this increase as indicative of a slow, gradual reduction and fixation of uranium upon burial in the reduced zone.

In summary, organic material is instrumental in the formation of the anoxic environment necessary for the preservation of, if not for the extraction of uranium from sea water, and organic material is able to receive uranium by adsorption.

Role of Phosphorus

The conditions of upwelling, high organic productivity, and accumulation of organic material that favor formation of uranium-enriched sediments also favor formation of phosphorite. The affinity of uranium for phosphate minerals is generally conceded, although Gavshin, Bobrov, and Zorkina $(1974$, p. 743$)$ claimed that the correlation holds only for phosphates formed under certain circumstances and is not general. The uranium in phosphorite is believed to be in carbonate fluorapatite, where tetravalent uranium probably substitutes for $\mathrm{Ca}^{+2}$ in the apatite crystal lattice, and hexavalent uranium is chemically adsorbed on the mineral by surface reactions of uranyl ions (Cullen, 1978, p. M67). Organisms extract phosphorus from the surface waters and carry it to the sea floor when they die and sink to the bottom, but if extraction of phosphorus by organisms is not accompanied by extraction of uranium, then the common correlation of phosphorus and uranium in marine sediments must be the result of interaction of the two elements after death of the organisms (Kuznetsov and others, 1968, p. 310). Thus, the concentration of uranium in sea-bed phosphorites seems mainly syngenetic with respect to phosphorus (Baturin and Kochenov, 1974, p. 99). The uranium content of biogenic phosphate material incorporated in diatom ooze is sometimes appreciably higher than the uranium content of the enclosing sediments; nevertheless, the uranium in the phosphate accounts for no more than 5-10 percent of the total uranium in bulk samples of the ooze; most of the uranium is in some way related to organic matter (Baturin and others, 1972, p. 283).

Veeh, Calvert, and Price (1974, p. 196) found that bulk analyses of diatomaceous sediments from off southwest Africa showed no clear correlation between uranium and phosphate. They concluded from this that if the fixation of uranium in sediments is controlled by the presence of phosphorus, then the uranium content must be controlled primarily by a particular phosphate mineral-presumably apatite-that contains variable amounts of uranium. Another possibility is that the lack of correlation of uranium and phosphorus in marine sediments is due to an absence of biogenic phosphorus in the sediments (Baturin and Yemel'yanov, 1973, p. 678).

Thus, although phosphorus is one possible agent for the extraction of uranium from sea water, it is by no means the only agent and probably is not the most important one.

Role of Silica

The possibility that part of the uranium found in the Monterey Formation is adsorbed onto, or otherwise involved with the opal of diatom frustules cannot be dismissed. Lewin (1961, p. 194) found that under particular conditions metal ions combine with the silica of diatom walls. Starik and Kolyadin (1957, p. 255) concluded that, under proper conditions, uranium in sea water exists as a hydrolyte that can be adsorbed on silica-bearing colloidal particles and is deposited on the sea bottom with these particles. Hurd (1973, p. 2275) pointed out that because of a strong negative charge on the surface of biogenic amorphous silica, the $\mathrm{UO}_{2}{ }^{+2}$ ion can be strongly adsorbed on the diatom walls. Little information concerning the possible role of opal in extraction of uranium from sea water is available, but uranium enrichment of 
opal and cristobalite does occur-although under circumstances different from those affecting the Monterey Formation. Zielinski (1980) described uraniferous secondary opal and chalcedony in Wyoming that formed from uraniferous, silica-saturated ground water. He proposed that both silica and uranium were released during dissolution of rhyolite glass and uranium was incorporated in silica gel, possibly by adsorption of uranyl-silica-hydroxyl complexes; dehydration of the gel then trapped the uranium. Glagolev $(1964$, p. 109) reported that cristobalite containing $1640 \mathrm{ppm}$ uranium was evenly distributed in a hypogene alteration product from previously deposited uraniferous opal.

\section{Diatomaceous Sediments}

A considerable amount of data on the uranium content of marine sediments is available. The average uranium content of 742 samples listed by Baturin $(1973$, p. 1033) was $3.7 \mathrm{ppm}$, and if landlocked basins are excluded, the average for 516 samples was $3.0 \mathrm{ppm}$. The uranium content of upper Quaternary marine sediments and of the surface layer of oceanic sediments ranged from 0.1 to $10 \mathrm{ppm}$ (Baturin, 1973, p. 1034). Kuznetsov, Simonyak, Lisitsyn, and Frenklikh (1968, p. 308) listed the uranium content of 57 samples of the surface layer of ocean sediments and found a range of $0.1-2.76 \mathrm{ppm}$, an average of $0.86 \mathrm{ppm}$, and a median value of $0.80 \mathrm{ppm}$. For comparison, the average uranium content of all shale has been estimated to be 3-4 ppm (Holland and Kulp, 1954, p. 203; Adams and others, 1959, table 7; Swanson, 1960, p. 4), about the same as for the outer few kilometers of the Earth's continental crust (Adams and others, 1959, p. 311). Baturin (1968b, p. 206) noted that the concentrations of metals that migrate only in suspension are relatively high in sediments of littoral zones, but the concentrations of metals that migrate in dissolved form, as does uranium, are relatively high in pelagic sediments. Boulad and Michard (1976, p. 82) studied cores from the southeast Atlantic Ocean that had oxidized sediments above reduced sediments, and they found the uranium content of the reduced sediments and their pore water to be greater than that of the oxidized sediments and their pore water. In landlocked seas uranium generally is concentrated in sediments in the centers of basins, but in the open ocean the sediments with the highest uranium content are on shelves and continental slopes (Baturin and Yemel'yanov, 1973, p. 674). In the open-shelf seas, uranium tends to be concentrated in the fine-grained sediments of deep basins (Baturin, 1973, p. 1033). Spalding, Exner, and McKee (1973) found in cores from an anoxic basin that the dissolved uranium and dissolved organic-carbon concentrations of pore water showed an increase with depth in the core, although the concentrations of both in the sediments remained nearly constant.
Some, but not all, diatomaceous sediments have higher uranium content than the average for marine sediments. Veeh, Calvert, and Price (1974, p. 194, 200) reported that organic-rich diatomaceous sediments on the continental margin of southwest Africa contain about an order of magnitude more uranium than do ordinary terrigenous marine sediments-from 10.2 to $54.6 \mathrm{ppm}$ uranium in cores, with higher concentrations at depth than at the sea-floor surface. Von Backstrom (1974, p. 618) found that siliceous sediments in the same area contained 5-93 ppm uranium and averaged $21 \mathrm{ppm}$. Baturin (1971a, p. 224) reported uranium concentrations in the interstitial water of diatomaceous ooze there of 1.3-650 ppb, and he noted that the ooze itself is characterized by uranium enrichment. Baturin considered the uranium in the interstitial water as mobile and capable of redistribution both vertically and horizontally, but he pointed out (1971a, p. 225) that diffusion of uranium into bottom water from diatomaceous ooze of the shelf and continental slope does not occur as it does from oxidized pelagic sediments. In shallow water where wave action aerates bottom water, uranium accumulates in the sediments to a lesser degree than it does under anoxic conditions (Baturin and others, 1972, p. 282-283).

The form of the uranium in marine sediments is in doubt. Veeh, Calvert, and Price (1974, p. 200) suggested that hexavalent uranium of sea water enters sediments by way of pore water and then is reduced to tetravalent uranium and incoroporated into carbonate fluorapatite growing diagenetically in the sediment. During a study of fission tracks of cores they found $(1974$, p. 193, 196) that uranium occurs off southwest Africa in diatomaceous mud as two principal modes: (1) finely and uniformly dispersed, and (2) in clusters, some of which could be traced to organic detritus or to small grains of phosphorite.

\section{Summary}

Several aspects of the occurrence of uranium in the marine environment are pertinent to an understanding of uranium in the Monterey Formation:

(1) Sea water is and has long been an adequate source for most of the uranium found in marine sediments that are enriched in uranium.

(2) Much of the uranium in sediments is connected in some way with organic material.

(3) The association of uranium and organic material begins, not with living organisms, and probably not during the sinking of dead organisms, but after the organic material is on the bottom, and perhaps partly after the organic material is buried.

(4) The principal process by which uranium is extracted from sea water most likely involves adsorption rather than chemical precipitation. 
(5) Anoxic conditions are conducive to the preservation of, if not to the formation of uranium-rich deposits.

(6) Some, but not all, richly diatomaceous sediments have uranium content higher than the average for all sediments.

(7) The uranium content of diatomaceous sediments can be greater below the surface than at the surface, and the uranium content of pore water can be considerably higher than that of sea water.

(8) Phosphatic material extracts uranium from sea water and adds to the total uranium content of diatomaceous sediments, but it is not the principal agent for uranium enrichment of most of the sediments.

\section{CONCLUSIONS}

The uranium in the Monterey Formation was derived chiefly from sea water at or near the time the Monterey sediments were deposited. Anoxic conditions associated with the accumulation of rich organic sediments certainly were conducive to the preservation of uranium-rich deposits, and perhaps to their formation. Except for certain secondary uranium minerals obviously related to vadose waters moving through rocks of the Monterey, the form of the uranium in the formation is uncertain. Most likely, the uranium is associated with organic matter, either by adsorption on organic material or by incorporation in organic complexes. Some uranium might be adsorbed on the biogenous opal of diatom frustules. During diagenesis of porcelanite, uranium probably is made available in a mobile form for enrichment of other rocks; chert, the end product of the diagenesis, has little uranium. The Monterey Formation, and similar rocks elsewhere, generally have more uranium than do most other kinds of rock. Within the Monterey, the cherty and dolomitic rocks, and the rocks having a high proportion of terrigenous clastic material generally have the lowest uranium content.

The uranium in the Monterey Formation apparently will have little commercial interest unless extraction of uranium from large volumes of low-grade material becomes economically feasible. However, a Monterey terrain could be a source of uranium for sandstone-type uranium deposits in other units. For example, uranium reported in the Sespe Formation near Ojai, California, could well have migrated from the Monterey.

\section{REFERENCES CITED}

Adams, J. A. S., Osmond, J. K., and Rogers, J. J. W., 1959, The geochemistry of thorium and uranium, Chapter 6 in Physics and Chemistry of the Earth, Volume 3: London, New York, Paris, Los Angeles, Pergamon Press, p. 298-348.
Adams, J. A. S., and Weaver, C. E., 1958, Thorium-to-uranium ratios as indicators of sedimentary processes-Example of concept of geochemical facies: American Association of Petroleum Geologists Bulletin, v. 42, no. 2, p. 387-430.

Arnal, R. E., and Vedder, J. G., 1976, Late Miocene paleobathymetry of California Continental Borderland north of $32^{\circ}$ [abs.]: American Association of Petroleum Geologists Bulletin, v. 60, no. 12, p. 2174.

Balistrieri, L., Brewer, P. G., and Murray, J. W., 1981, Scavenging residence times of trace metals and surface chemistry of sinking particles in the deep ocean: Deep-Sea Research, v. 28A, p. 101-121.

Bandy, O. L., and Arnal, R. E., 1969, Middle Tertiary basin development, San Joaquin Valley, California: Geological Society of America Bulletin, v. 80, no. 5, p. 783-819.

Bandy, O. L., and Kolpack, R. L., 1963, Foraminiferal and sedimentological trends in the Tertiary section of Tecolote Tunnel, California: Micropaleontology, v. 9, no. 2, p. 117-170.

Barron, J. A., 1973, Late Miocene-early Pliocene paleotemperatures for California from marine diatom evidence: Palaeogeography, Palaeoclimatology, Palaeoecology, v. 14 , no. 4 , p. $277-291$.

1975, Late Miocene-early Pliocene marine diatoms from southern California: Palaeontographica, Abt. B, v. 151, p. 97-170.

Baturin, G. N. 1968a, Geochemistry of uranium in the Baltic: Geochemistry International, v. 5, no. 2, p. 344-348.

$1968 \mathrm{~b}$, Proportion of the forms of uranium migration in water from certain USSR rivers: Doklady Akademii Nauk SSSR, v. 178, p. 206-209.

1969, Uranium in the surface layer of sediments of the northwestern Indian Ocean: Oceanology, v. 9, no. 6, p. 828-833.

1971a, Uranium in oceanic ooze solutions of the southeastern Atlantic: Doklady Akademii Nauk SSSR 1971, v. 198, p. 224-226.

1971b, Formation of phosphate sediments and water dynamics: Oceanology, v. 11, no. 3, p. 372-376.

1973, Uranium in the modern marine sedimentary cycle: Geochemistry International, v. 10, no. 5, p. 1031-1041. 1974, Average ratios of uranium and organic matter in Holocene sea and ocean sediments: Doklady Akademii Nauk SSSR 1974, v. 207, p. 190-192.

Baturin, G. N., and Kochenov, A. V., 1973, Uranium in the interstitial waters of marine and oceanic sediments [abs.]: Geochemistry International, v. 10, no. 5, p. 1169.

1974, Uranium content of oceanic phosphorites: Lithology and Mineral Resources, v. 9, no. 1, p. 99-103.

Baturin, G. N., Kochenov, A. V., and Kovaleva, S. A., 1966, Some features of uranium distribution in Black Sea water: Doklady Akademii Nauk SSSR, v. 166, p. 172-174.

Baturin, G. N., Kochenov, A. V., and Senin, Y. M., 1972, Uranium concentration in Recent ocean sediments in zones of rising currents: Geochemistry International, v. 8, no. 2, p. 281-286.

Baturin, G. N., Lebedev, L. I., and Mayev, Y. G., 1976, Uranium in Caspian Sea sediments: Oceanology, v. 15, no. 1, p. 87-91. 
Baturin, G. N., and Lisitsyn, A. P., 1973, Uranium in the Bering Sea sediments: Oceanology, v. 13, no. 6, p. 876-881.

Baturin, G. N., and Yemel'yanov, Y. M., 1973, Uranium distribution in Mediterranean sedments: Oceanology, v. 13, no. 5, p. 674-679.

Bell, K. G., 1960, Uranium and other trace elements in petroleums and rock asphalts: U.S. Geological Survey Professional Paper 356-B, p. 45-65.

Berner, R. A., 1978, Sulfate reduction and the rate of deposition of marine sediments: Earth and Planetary Science Letters, v. 37, no. 3, p. 492-498.

Bloch, Salman, 1980, Some factors controlling the concentration of uranium in the world ocean: Geochimica et Cosmochimica Acta, v. 44, no. 2, p. 373-378.

Bonatti, Enrico, Fisher, D. E., Joensuu, Oiva, and Rydell, H. S., 1971, Postdepositional mobility of some transition elements, phosphorus, uranium, and thorium in deep sea sediments: Geochimica et Cosmochimica Acta, v. 35, no. 2, p. 189-201.

Boon, J. J., De Leeuw, J. W., and Burlingame, A. L., 1978, Organic geochemistry of Walvis Bay diatomaceous oozeIII. Structural analysis of the monoenoic and polycyclic fatty acids: Geochimica et Cosmochimica Acta, v. 42, no. 6, p. 631-644.

Boulad, A. P., and Michard, Gil, 1976, Etude de l'uranium, du thorium et de leurs isotopes dan quelques carottes du Bassin Anagolais (Atlantique Sud-Est): Earth and Planetary Science Letters, v. 32, no. 1, p. 77-83.

Bowes, W. A., 1955, Uranium in the Kern County area [abs.]: Pacific Petroleum Geologist, v. 9, no. 5, p. 2-3.

Bramlette, M. N., 1946, The Monterey Formation of California and the origin of its siliceous rocks: U.S. Geological Survey Professional Paper 212, $57 \mathrm{p}$.

Brongersma-Sanders, Margaretha, 1948, The importance of upwelling water to vertebrate paleontology and oil geology: Verhandelingen der Koniklijke Nederlandsche Akademie van Wetenschappen, Afdeeling Naturrkunde (Tweede Sectie), v. 45 , no. 4 , p. 1-112.

1951, On conditions favouring the preservation of chlorophyll in marine sediments, in World Petroleum Congress, Third, The Hague, 1951, Proceedings, Section I, Geology, Geophysics: Leiden, E. J. Brill, p. 401-413.

1957, Mass mortality in the sea, Chapter 29 in Treatise on marine ecology and Paleocology, Volume 1, Ecology: Geological Society of America Memoir 67, p. 941-1010.

1966, The fertility of the sea and its bearing on the origin of oil: The Advancement of Science, v. 23, no. 107, p. 41-46.

Burton, J. D., 1965, Radioactive nuclides in sea water, marine sediments, and marine organisms, Chapter 22 in Chemical Oceanography, Volume 1: London and New York, Academic Press, p. 425-475.

1975, Radioactive nuclides in the marine environment, Chapter 18 in Chemical Oceanography, Volume 3, [2d ed.]: London, New York, San Francisco, Academic Press, p. 91-191.

Burton, V. L., and Sullivan, G. R., 1951, Carbon content and radioactivity of marine rocks: American Geophysical Union Transactions, v. 32, no. 6, p. 881-884.
Busch, W. H., and Keller, G. H., 1981, The physical properties of Peru-Chile continental margin sediments-The influence of coastal upwelling on sediment properties: Journal of Sedimentary Petrology, v. 51, no. 3, p. 705-719.

Calvert, S. E., 1966, Accumulation of diatomaceous silica in the sediments of the Gulf of California: Geological Society of America Bulletin, v. 77, no. 6, p. 569-596.

1974, Deposition and diagenesis of silica in marine sediments, in Pelagic sediments, on land and under the sea: International Association of Sedimentologists Special Publication 1, p. 273-299.

Casey, R. E., and Price, A. B., 1973, A tentative radiolarian zonation and paleo-oceanographic interpretation from Newport Bay, California, in Miocene sedimentary environments and biofacies, southeastern Los Angeles basin: Society of Economic Paleontologists and Mineralogists, Annual Meeting 1973, Trip 1, p. 67-70.

Cornell, W. C., 1969, Silicoflagellates as paleoenvironment indicators in the Modelo Formation (Miocene): Geological Society of America Abstracts with Programs, 1969, pt. 2, p. 6-7.

Cullen, D. J., 1978, The uranium content of submarine phosphorite and glauconite deposits on Chatham Rise, east of New Zealand: Marine Geology, v. 28, no. 3-4, p. M67-M78.

David, L. R., 1940a, Miocene fishes in well cores from Torrance in southern California: American Association of Petroleum Geologists Bulletin, v. 24, no. 12, p. 2182-2184. $1940 \mathrm{~b}$, Upper Miocene fish from northern rim of the Santa Monica Mountains, California [abs.]: Geological Society of America Bulletin, v. 51, no. 12, pt. 2, p. 1982-1983.

1943, Miocene fishes of southern California: Geological Society of America Special Paper 43, 193 p.

Degens, E. T., Khoo, Francis, and Michaelis, Walter, 1977, Uranium anomaly in Black Sea sediments: Nature, v. 269, no. 5629 , p. 566-569.

Durham, D. L., 1974, Geology of the southern Salinas Valley area, California: U.S. Geological Survey Professional Paper 819, $111 \mathrm{p}$.

Finch, W. I., 1967, Geology of epigenetic uranium deposits in sandstone in the United States: U.S. Geological Survey Professional Paper 538, 121 p.

Gavshin, V. M., Bobrov, V. A., and Zorkina, L. S., 1974, quantitative relations between uranium and phosphorus in phosphorites and phosphatic sedimentary rocks: Lithology and Mineral Resources, v. 9, no. 6, p. 740-746.

Glagolev, N. A., 1964, Uraniferous cristobalite and tridymite from limestone: Doklady Akademii Nauk SSSR, v. 143, p. 108-109.

Goldberg, E. D., 1963, The oceans as a chemical system, in The Sea, Volume 2: New York, Interscience Publishers, p. 3-25.

Hail, W. J., Jr., Myers, A. T., and Horr, C. A., 1956, Uranium in asphalt-bearing rocks of the western United States: U.S. Geological Survey Professional Paper 300, p. 521-526.

Hein, J. R., Scholl, D. W., Barron, J. A., Jones, M. G., and Miller, Jacquelyn, 1978, Diagenesis of late Cenozoic diatomaceous deposits and formation of the bottom simulating reflector in the southern Bering Sea: Sedimentology, v. 25 , no. 2 , p. 155-181. 
Hodge, V. F., Koide, M., and Goldberg, E. D., 1979, Particulate uranium, plutonium, and polonium in the biogeochemistries of the coastal zone: Nature, v. 277, no. 5693, p. 206-209.

Holland, H. D., 1979, Metals in black shales-A reassessment: Economic Geology, v. 74, no. 7, p. 1676-1680.

Holland, H. D., and Kulp, J. L., 1954, The transport and deposition of uranium, ionium and radium in rivers, oceans, and ocean sediments: Geochimica et Cosmochimica Acta, v. 5, no. 5, p. 197-213.

Hurd, D. C., 1973, Interactions of biogenic opal, sediment and seawater in the central equatorial Pacific: Geochimica et Cosmochimica Acta, v. 37, p. 2257-2282.

Iijima, Azuma, and Tada, Ryuji, 1981, Silica diagenesis of Neocene diatomaceous and volcaniclastic sediments in northern Japan: Sedimentology, v. 28, no. 2, p. 185-200.

Ingle, J. C., Jr., 1963a, Paleoecologic, sedimentary, and structural history of the late Tertiary Capistrano embayment, California [abs.]: American Association of Petroleum Geologists Bulletin, v. 47, no. 2, p. 361.

$1963 \mathrm{~b}$, Miocene-Pliocene paleoecology of San Fernando basin, California [abs.]: American Association of Petroleum Geologists Bulletin, v. 47, no. 9, p. 1771-1772. 1967 , Foraminiferal biofacies variation and the MiocenePliocene boundary in southern California: Bulletins of American Paleontology, v. 52, no. 236, p. 217-394.

1972, Biostratigraphy and paleoecology of early Miocene through early Pleistocene benthonic and planktonic Foraminifera, San Joaquin Hills-Newport Bay, Orange County, California, in The Pacific Coast Miocene Biostratigraphic Symposium, Proceedings: Society of Economic Paleontologists and Mineralogists, Pacific Section, p. $255-283$.

1973, Biostratigraphy and paleoecology of early Miocene through early Pleistocene benthonic and planktonic Foraminifera, San Joaquin Hills-Newport Bay-Dana Point area, Orange County, California, in Miocene sedimentary environments and biofacies, southeastern Los Angeles basin: Society of Economic Paleontologists and Mineralogists, Annual Meeting, 1973, Trip 1, p. 18-38.

Issacs, C. M., 1979, Lateral diagenesis in Monterey Shale, Santa Barbara coast, California [abs.]: American Association of Petroleum Geologists Bulletin, v. 63, no. 3, p. 473.

Kastner, M., Keene, J. B., and Gieskes, J. M., 1977, Diagenesis of siliceous oozes-I. Chemical controls on the rate of opalA to opal-CT transformation-An exerimental study: Geochimica et Cosmochimica Acta, v. 41, p. 1041-1059.
Kleinpell, R. M., 1938, Miocene stratigraphy of California Tulsa, Oklahoma, American Association of Petroleum Geologists, $450 \mathrm{p}$.

Kochenov, A. V., Korolev, K. G., Dubinchuk, V. T., and Medvedcv, Y. L., 1977, Experimental data on the conditions of precipitation of uranium from aqueous solutions: Geochemistry International, v. 14, no. 4, p. 82-87.

$\mathrm{Ku}$, Teh-Lung, Knauss, K. G., and Mathieu, G. G., 1977, Uranium in open ocean-Concentration and isotopic compositions: Deep-Sea Research, v. 24, no. 11, p. 1005-1017.

Kuznetsov, Y. A., Simonyak, Z. N., Lisitsyn, A. P., and Frenklikh, M. S., 1968, Uranium and radium in the surface layer of oceanic sediments: Geochemistry International, v. 5, no. 2, p. 306-313.

Langmuir, Donald, 1977, Uranium solution-mineral equilibria at low temperatures with applications to sedimentary ore deposits: U.S. Department of Energy Report GJO-1659-3, $39 \mathrm{p}$.

1978, Uranium solution-mineral equilibria at low temperatures with applications to sedimentary ore deposits: Geochimica et Cosmochimica Acta, v. 42, no. 6, p. 547-569.

Lewin, J. C., 1961, The dissolution of silica from diatom walls: Geochimica et Cosmochimica Acta, v. 21, nos. 3-4, p. 182-198.

Lipps, J. H., and Kalisky, Maurice, 1973, Calcareous nannoplankton biostratigraphy and paleoecology in OligoMiocene of California [abs.]: American Association of Petroleum Geologists Bulletin, v. 57, no. 2, p. 436.

Lisitsyn, A. P., 1967, Basic relationships in distribution of modern siliceous sediments and their connection with climatic zonation: International Geology Review, v. 9, no. 5, p. 631-652; no. 6, p. 842-865; no. 7, p. 980-1004; no. 8, p. $1114-1130$.

1972, Sedimentation in the World Ocean: Society of Economic Paleontologists and Mineralogists Special Publication no. 17, $218 \mathrm{p}$.

Mandra, Y. T., 1959, Use of fossil silicoflagellates in paleogeography [abs.]: Geological Society of America Bulletin, v. 70, no. 12 , pt. 2, p. 1734.

Mo, T., Suttle, A. D., and Sackett, W. M., 1971, Uranium concentration in marine sediments [abs.]: Eos (American Geophysical Union Transactions), v. 52, no. 4, p. 361 .

Muller, P. J., and Suess, E., 1979, Productivity, sedimentation rate, and sedimentary organic matter in the oceansI. Organic carbon preservation: Deep-Sea Research, v. 26, no. $12 \mathrm{~A}$, p. 1347-1362. 
Murata, K. J., Friedman, Irving, and Gleason, J. D., 1977, Oxygen isotope relations between diagenetic silica minerals in Monterey Shale, Temblor Range, California: American Journal of Science, v. 277, no. 3, p. 259-272.

Murata, K. J., and Larson, R. R., 1975, Diagenesis of Miocene siliceous shales, Temblor Range, California: U.S. Geological Survey Journal of Research, v. 3, no. 5, p. 553-556.

Murata, K. J., and Nakata, J. K., 1974, Cristobalitic stage in the diagenesis of diatomaceous shale: Science, v. 184, no. 4136, p. 567-568.

Osterwald, F. W., 1965, Structural control of uranium-bearing vein deposits and districts in the conterminous United States: U.S. Geological Survey Professional Paper 455-G, p. 121-146.

Patet, Alix, 1972, A subsurface study of the foraminiferal fauna of the Vaqueros, the Rincon, and the lower Monterey Formations from the Elwood oil field in Santa Barbara County, California, in The Pacific Coast Miocene Biostratigraphic Symposium, Proceedings: Society of Economic Paleontologists and Mineralogists, Pacific Section, p. 150-157.

Pierce, R. L., 1956, Upper Miocene Foraminifera and fish from the Los Angeles area, California: Journal of Paleontology, v. 30, no. 6, p. 1288-1314.

Pokidin, V. K., Kuznetsov, Y. V., Prozorovich, E. A., and Asadullayeva, F. A., 1972, Radioactivity of the Caspian Sea sediments and their deposition rates: Geochemistry International v. 9, no. 4, p. 567-575.

Ramsay, A. T. S., 1973, A history of organic siliceous sediments in oceans, in Organisms and continents through time: The Paleontological Association, Special Papers in Paleontology, no. 12, p. 199-234.

Redfield, A. C., Ketchum, B. H., and Richards, F. A., 1963, The influence of organisms on the composition of sea-water, in The Sea, Volume 2: New York, Interscience Publishers, p. 26-77.

Ross, V. F., 1952, Autoradiographic study of marine shales: Economic Geology, v. 47, no. 8, p. 783-793.

Schrader, Hans-Joachim, 1971, Fecal pellets-Role in sedimentation of pelagic diatoms: Science, v. 174, no. 4004, p. 55-57.

Siesser, W. G., 1980, Late Miocene origin of the Benguela upwelling system off northern Namibia: Science, v. 208, p. 283-285.

Siever, Raymond, and Stein, C. L., 1976, Models for the transformation of biogenic silica to chert [abs.]: Eos (American Geophysical Union Transactions), v. 57, no. 4, p. 256.
Smayda, T. J., 1971, Normal and accelerated sinking of phytoplankton in the sea: Marine Geology, v. 11, no. 2, p. 105-122.

Smith, P. B., 1968, Paleoenvironment of phosphate-bearing Monterey Shale in Salinas Valley, California: American Association of Petroleum Geologists Bulletin, v. 52, no. 9, p. 1785-1791.

Spalding, R. F., Exner, M. E., and McKee, T. R., 1973, Uranium geochemistry at the sediment-water interface of an anoxic basin [abs.]: Eos (American Geophysical Union Transactions), v. 54, no. 4, p. 341.

Starik, I. E. and Kolyadin, L. B., 1957, The occurrence of uranium in ocean water: Geochemistry No. 3, p. 245-256.

Swanson, V. E., 1960, Oil yield and uranium content of black shales: U.S. Geological Survey Professional Paper 356-A, p. 1-44.

1961, Geology and geochemistry of uranium in marine black shales-A review: U.S. Geological Survey Professional Paper 356-C, p. 67-112.

Troxel, B. W., and Morton, P. K., 1962, Mines and mineral resources of Kern County, California: California Division of Mines and Geology, County Report 1, $370 \mathrm{p}$.

Troxel, B. W., Stinson, M. C., and Chesterman, C. W., 1957, Uranium, in Mineral commodities of California: California Division of Mines Bulletin 176, p. 669-687.

Veeh, H. H., 1967, Deposition of uranium from the ocean: Earth and Planetary Science Letters, v. 3, p. 145-150.

Veeh, H. H. Calvert, S. E., and Price, N. B., 1974, Accumulation of uranium in sediments and phosphorites on the south west African shelf: Marine Chemistry, v. 2, no. 3, p. 189-202.

Von Backstrom, J. W., 1974, Other uranium deposits, in Formation of uranium ore deposits; Symposium on formation of uranium ore deposits, Athens, Greece, 6-10 May, 1974: Vienna, International Atomic Energy Agency, p. 605-624.

Walker, G. W., Lovering, T. G., and Stephens, H. G., 1956, Radioactive deposits in California: California Division of Mines Special Report 49, 38 p.

Weber, F. F., Jr., and Sackett, W. M., 1981, Uranium geochemistry of Orca Basin: Geochimica et Cosmochimica Acta, v. 45, no. 8, p. 1321-1329.

Zielinski, R. A., 1980, Uranium in secondary silica-A possible exploration guide: Economic Geology, v. 75, no. 4, p. 592-602.

Zverev, V. L., Spiridonov, A. I., and Shvets V. M., 1976, On the balance of uranium in the ocean: Geochemistry International, v. 13 , no. 3 , p. 88-93. 




